\title{
Decomposition Formulas for Triple $q$-Hypergeometric Functions
}

\author{
Thomas Ernst \\ Department of Mathematics, Uppsala University, P.O. Box 480, SE-751 06 Uppsala, Sweden \\ Correspondence should be addressed to Thomas Ernst; thomas@math.uu.se
}

Received 11 November 2013; Accepted 31 December 2013; Published 15 May 2014

Academic Editor: R. Yuster

Copyright (C) 2014 Thomas Ernst. This is an open access article distributed under the Creative Commons Attribution License, which permits unrestricted use, distribution, and reproduction in any medium, provided the original work is properly cited.

In the spirit of Hasanov, Srivastava, and Turaev (2006), we introduce new inverse operators together with a more general operator and find a summation formula for the last one. Based on these operators and the earlier known $q$-analogues of the BurchnallChaundy operators, we find 15 symbolic operator formulas. Then, 10 expansions for the $q$-analogues of Srivastava's three triple hypergeometric functions in terms of ${ }_{4} \phi_{3} q$-hypergeometric and $q$-Kampé de Fériet functions are derived. These expansions readily reduce to 10 new expansions for the three triple Srivastava hypergeometric functions in terms of ${ }_{4} F_{3}$ hypergeometric and Kampé de Fériet functions.

\section{Introduction}

The concept of decomposition formulas for multiple hypergeometric functions is well known from the articles by Hasanov et al. [1] and Bin-Saad [2]. This paper follows the first one by using $q$-analogues of 15 symbolic operator formulas.

The paper is organized as follows. In Section 2, we give the basic definitions and introduce a new inverse $q$-analogue of the Burchnall-Chaundy operators for an arbitrary number of variables, together with an explicit summation formula for one of these; the proof uses a $q$-Lauricella function summation formula. In Section 3, we express the three triple Srivastava $q$-hypergeometric functions in terms of these operators and $q$-hypergeometric and $q$-Appell functions.

Finally, we find 10 expansions for these triple $q$-hypergeometric functions. The proofs show a certain symmetry, which is explained in detail in the last section.

\section{Definitions}

We start by defining the umbral notation [3], a mixture of Heine 1846 and [4].

Definition 1. The power function is defined by $q^{a} \equiv e^{\operatorname{alog}(q)}$. We always assume that $0<q<1$. Let $\delta>0$ be an arbitrary small number. We will use the following branch of the logarithm: $-\pi+\delta<\operatorname{Im}(\log q) \leq \pi+\delta$. This defines a simply connected space in the complex plane.
The variables $a, b, c, \ldots \in \mathbb{C}$ denote certain parameters. The variables $i, j, k, l, m, n$ will denote natural numbers except for certain cases where it will be clear from the context that $i$ will denote the imaginary unit. The $q$-shifted factorial is given by

$$
\langle a ; q\rangle_{n} \equiv \prod_{m=0}^{n-1}\left(1-q^{a+m}\right) ;(a ; q)_{n} \equiv \prod_{m=0}^{n-1}\left(1-a q^{m}\right) .
$$

Since products of $q$-shifted factorials occur so often, to simplify them, we will frequently use the more compact notation:

$$
\left\langle a_{1}, \ldots, a_{m} ; q\right\rangle_{n} \equiv \prod_{j=1}^{m}\left\langle a_{j} ; q\right\rangle_{n} .
$$

The operator

$$
\sim: \frac{\mathbb{C}}{\mathbb{Z}} \longmapsto \frac{\mathbb{C}}{\mathbb{Z}}
$$

is defined by

$$
a \longmapsto a+\frac{\pi i}{l \log q} .
$$

By (4), it follows that

$$
\widetilde{\langle a ; q\rangle_{n}}=\prod_{m=0}^{n-1}\left(1+q^{a+m}\right) .
$$


Assume that $(m, l)=1$; that is, $m$ and $l$ relatively prime. The operator

$$
\frac{\widetilde{m}}{l}: \frac{\mathbb{C}}{\mathbb{Z}} \longmapsto \frac{\mathbb{C}}{\mathbb{Z}}
$$

is defined by

$$
a \longmapsto a+\frac{2 \pi i m}{l \log q}
$$

Furthermore,

$$
\begin{gathered}
(a ; q)_{\infty}=\prod_{m=0}^{\infty}\left(1-a q^{m}\right), \quad 0<|q|<1, \\
(a ; q)_{\alpha} \equiv \frac{(a ; q)_{\infty}}{\left(a q^{\alpha} ; q\right)_{\infty}}, \quad a \neq q^{-m-\alpha}, m=0,1, \ldots
\end{gathered}
$$

The $q$-gamma function is given by

$$
\Gamma_{q}(z) \equiv \frac{\langle 1 ; q\rangle_{\infty}}{\langle z ; q\rangle_{\infty}}(1-q)^{1-z}, \quad 0<|q|<1 .
$$

To save space, the following notation for quotients of $\Gamma_{q}$ functions will often be used:

$$
\Gamma_{q}\left[\begin{array}{c}
a_{1}, \ldots, a_{p} \\
b_{1}, \ldots, b_{r}
\end{array}\right] \equiv \frac{\Gamma_{q}\left(a_{1}\right) \cdots \Gamma_{q}\left(a_{p}\right)}{\Gamma_{q}\left(b_{1}\right) \cdots \Gamma_{q}\left(b_{r}\right)} .
$$

On the ring of polynomials $\mathbb{C}[x]$, we define the functions $\epsilon_{i}$ : $\mathbb{C}[x] \mapsto \mathbb{C}[x]$ by

$$
\left[\epsilon_{i}\right] f\left(x_{i}\right) \equiv f\left(q x_{i}\right) .
$$

The following notation is often used when we have long exponents:

$$
\mathrm{QE}(x) \equiv q^{x} .
$$

The following notation will sometimes be used:

$$
\widehat{a} \equiv a \vee \widetilde{a} \vee^{\widetilde{m / n} a}
$$

Definition 2. Generalizing Heine's series, we will define a $q$ hypergeometric series by

$$
\begin{aligned}
& p+p^{\prime} \phi_{r+r^{\prime}}\left[\begin{array}{lll}
\widehat{a}_{1}, \ldots, \widehat{a}_{p} & \prod_{i} f_{i}(k) \\
\widehat{b}_{1}, \ldots, \widehat{b}_{1} & \mid q ; z \| & \prod_{i} g_{i}(k)
\end{array}\right] \\
& \equiv \sum_{k=0}^{\infty} \frac{\left\langle\widehat{a}_{1} ; q\right\rangle_{k} \cdots\left\langle\widehat{a}_{p} ; q\right\rangle_{k}}{\left\langle 1, \widehat{b}_{1} ; q\right\rangle_{k} \cdots\left\langle\widehat{b}_{r} ; q\right\rangle_{k}}\left[(-1)^{k} q^{\left.\left(\begin{array}{c}
k \\
2
\end{array}\right)\right]^{1+r+r^{\prime}-p-p^{\prime}}}\right. \\
& \times z^{k} \frac{\prod_{i} f_{i}(k)}{\prod_{i} g_{i}(k)} .
\end{aligned}
$$

We assume that the $f_{i}(k)$ and $g_{j}(k)$ contain $p^{\prime}$ and $r^{\prime}$ factors of the form $\langle\widehat{a(k)} ; q\rangle_{k}$ or $(s(k) ; q)_{k}$, respectively. The notation $\sum_{\vec{m}}$ denotes a multiple summation with the indices $m_{1}, \ldots, m_{n}$ running over all nonnegative integer values. In this connection, we put $m \equiv \sum_{j=1}^{n} m_{j}$.
Definition 3. The vectors

$$
(a),(b),\left(g_{i}\right),\left(h_{i}\right),\left(a^{\prime}\right),\left(b^{\prime}\right),\left(g_{i}^{\prime}\right),\left(h_{i}^{\prime}\right)
$$

have dimensions

$$
A, B, G, H, A^{\prime}, B^{\prime}, G^{\prime}, H^{\prime} \text {. }
$$

Let

$$
1+B+B^{\prime}+H+H^{\prime}-A-A^{\prime}-G-G^{\prime} \geq 0 .
$$

Then, the generalized $q$-Kampé de Fériet function is defined by

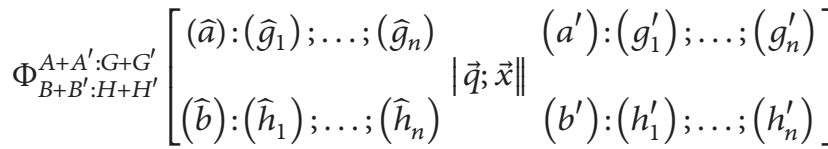

$$
\begin{aligned}
& \equiv \sum_{\vec{m}}\left(\left\langle(\widehat{a}) ; q_{0}\right\rangle_{m}\left(a^{\prime}\right)\left(q_{0}, m\right) \prod_{j=1}^{n}\left\langle\left(\widehat{g}_{j}\right) ; q_{j}\right\rangle_{m_{j}}\right. \\
& \left.\times\left(\left(g_{j}^{\prime}\right)\left(q_{j}, m_{j}\right) x_{j}^{m_{j}}\right)\right) \\
& \times\left(\left\langle(\widehat{b}) ; q_{0}\right\rangle_{m}\left(b^{\prime}\right)\left(q_{0}, m\right)\right. \\
& \left.\times \prod_{j=1}^{n}\left(\left\langle\left(\widehat{h}_{j}\right) ; q_{j}\right\rangle_{m_{j}}\left(h_{j}^{\prime}\right)\left(q_{j}, m_{j}\right)\left\langle 1 ; q_{j}\right\rangle_{m_{j}}\right)\right)^{-1} \\
& \times(-1)^{\sum_{j=1}^{n} m_{j}\left(1+H+H^{\prime}-G-G^{\prime}+B+B^{\prime}-A-A^{\prime}\right)} \\
& \times Q E\left(\left(B+B^{\prime}-A-A^{\prime}\right)\left(\begin{array}{c}
m \\
2
\end{array}\right), q_{0}\right) \\
& \times \prod_{j=1}^{n} Q E\left(\left(1+H+H^{\prime}-G-G^{\prime}\right)\left(\begin{array}{c}
m_{j} \\
2
\end{array}\right), q_{j}\right) .
\end{aligned}
$$

It is assumed that there are no zero factors in the denominator. We assume that

$$
\left(a^{\prime}\right)\left(q_{0}, m\right),\left(g_{j}^{\prime}\right)\left(q_{j}, m_{j}\right),\left(b^{\prime}\right)\left(q_{0}, m\right),\left(h_{j}^{\prime}\right)\left(q_{j}, m_{j}\right)
$$

contain factors of the form $\langle\widehat{a}(k) ; q\rangle_{k},(s ; q)_{k},(s(k) ; q)_{k}$, or $Q E(f(\vec{m}))$. In the rest of the paper, we write $\langle a ; q\rangle_{m}$ instead of $\langle\widehat{a} ; q\rangle_{m}$. 
Definition 4. The four $q$-Appell functions are given by

$$
\begin{aligned}
& \Phi_{1}\left(a ; b, b^{\prime} ; c \mid q ; x_{1}, x_{2}\right) \\
& \equiv \sum_{m_{1}, m_{2}=0}^{\infty} \frac{\langle a ; q\rangle_{m_{1}+m_{2}}\langle b ; q\rangle_{m_{1}}\left\langle b^{\prime} ; q\right\rangle_{m_{2}}}{\langle 1 ; q\rangle_{m_{1}}\langle 1 ; q\rangle_{m_{2}}\langle c ; q\rangle_{m_{1}+m_{2}}} x_{1}^{m_{1}} x_{2}^{m_{2}} \\
& \max \left(\left|x_{1}\right|,\left|x_{2}\right|\right)<1, \\
& \Phi_{2}\left(a ; b, b^{\prime} ; c, c^{\prime} \mid q ; x_{1}, x_{2}\right) \\
& \equiv \sum_{m_{1}, m_{2}=0}^{\infty} \frac{\langle a ; q\rangle_{m_{1}+m_{2}}\langle b ; q\rangle_{m_{1}}\left\langle b^{\prime} ; q\right\rangle_{m_{2}}}{\langle 1 ; q\rangle_{m_{1}}\langle 1 ; q\rangle_{m_{2}}\langle c ; q\rangle_{m_{1}}\left\langle c^{\prime} ; q\right\rangle_{m_{2}}} x_{1}^{m_{1}} x_{2}^{m_{2}}, \\
& \left|x_{1}\right|+\left|x_{2}\right|<1, \\
& \Phi_{3}\left(a, a^{\prime} ; b, b^{\prime} ; c \mid q ; x_{1}, x_{2}\right) \\
& \equiv \sum_{m_{1}, m_{2}=0}^{\infty} \frac{\langle a ; q\rangle_{m_{1}}\left\langle a^{\prime} ; q\right\rangle_{m_{2}}\langle b ; q\rangle_{m_{1}}\left\langle b^{\prime} ; q\right\rangle_{m_{2}}}{\langle 1 ; q\rangle_{m_{1}}\langle 1 ; q\rangle_{m_{2}}\langle c ; q\rangle_{m_{1}+m_{2}}} x_{1}^{m_{1}} x_{2}^{m_{2}}, \\
& \max \left(\left|x_{1}\right|,\left|x_{2}\right|\right)<1, \\
& \Phi_{4}\left(a ; b ; c, c^{\prime} \mid q ; x_{1}, x_{2}\right) \\
& \equiv \sum_{m_{1}, m_{2}=0}^{\infty} \frac{\langle a ; q\rangle_{m_{1}+m_{2}}\langle b ; q\rangle_{m_{1}+m_{2}}}{\langle 1 ; q\rangle_{m_{1}}\langle 1 ; q\rangle_{m_{2}}\langle c ; q\rangle_{m_{1}}\left\langle c^{\prime} ; q\right\rangle_{m_{2}}} x_{1}^{m_{1}} x_{2}^{m_{2}}, \\
& \left|\sqrt{x_{1}}\right|+\left|\sqrt{x_{2}}\right|<1 \text {. }
\end{aligned}
$$

The $q$-analogues of the Srivastava triple hypergeometric functions are

$$
\begin{aligned}
& H_{A}\left(a, b_{1}, b_{2} ; c_{1}, c_{2} \mid q ; x_{1}, x_{2}, x_{3}\right) \\
& \equiv \sum_{m, n, p=0}^{\infty} \frac{\langle a ; q\rangle_{m+p}\left\langle b_{1} ; q\right\rangle_{m+n}\left\langle b_{2} ; q\right\rangle_{n+p}}{\left\langle 1, c_{1} ; q\right\rangle_{m}\langle 1 ; q\rangle_{n}\langle 1 ; q\rangle_{p}\left\langle c_{2} ; q\right\rangle_{n+p}} x_{1}^{m} x_{2}^{n} x_{3}^{p}, \\
& H_{B}\left(a, b_{1}, b_{2} ; c_{1}, c_{2}, c_{3} \mid q ; x_{1}, x_{2}, x_{3}\right) \\
& \equiv \sum_{m, n, p=0}^{\infty} \frac{\langle a ; q\rangle_{m+p}\left\langle b_{1} ; q\right\rangle_{m+n}\left\langle b_{2} ; q\right\rangle_{n+p}}{\left\langle 1, c_{1} ; q\right\rangle_{m}\left\langle 1, c_{2} ; q\right\rangle_{n}\left\langle 1, c_{3} ; q\right\rangle_{p}} x_{2}^{n} x_{3}^{p}, \\
& H_{C}\left(a, b_{1}, b_{2} ; c \mid q ; x_{1}, x_{2}, x_{3}\right) \\
& \equiv \sum_{m, n, p=0}^{\infty} \frac{\langle a ; q\rangle_{m+p}\left\langle b_{1} ; q\right\rangle_{m+n}\left\langle b_{2} ; q\right\rangle_{n+p}}{\langle 1 ; q\rangle_{m}\langle 1 ; q\rangle_{n}\langle 1 ; q\rangle_{p}\langle c ; q\rangle_{m+n+p}} x_{1}^{m} x_{2}^{n} x_{3}^{p} .
\end{aligned}
$$

Definition 5. In the spirit of Burchnall and Chaundy [5], the following inverse pair of symbolic operators was introduced in [3]; compare Jackson [6]. Let

$$
\theta_{q, j} \equiv x_{j} D_{q, x_{j}}
$$

Then,

$$
\begin{aligned}
& \nabla_{x_{1}, x_{2} ; q}(h) \equiv \Gamma_{q}\left[\begin{array}{l}
h, h+\theta_{q, 1}+\theta_{q, 2} \\
h+\theta_{q, 1}, h+\theta_{q, 2}
\end{array}\right], \\
& \Delta_{x_{1}, x_{2} ; q}(h) \equiv \Gamma_{q}\left[\begin{array}{l}
h+\theta_{q, 1}, h+\theta_{q, 2} \\
h+\theta_{q, 1}+\theta_{q, 2}, h
\end{array}\right] .
\end{aligned}
$$

We limit our considerations to the case $h \neq-n$.

Then, one has

$$
\begin{gathered}
\nabla_{x_{1}, x_{2} ; q}(h)\langle h ; q\rangle_{m}\langle h ; q\rangle_{n} x_{1}^{m} x_{2}^{n}=\langle h ; q\rangle_{m+n} x_{1}^{m} x_{2}^{n}, \\
\nabla_{x_{1}, x_{2} ; q}(h)=\sum_{r=0}^{\infty} \frac{\left\langle-\theta_{q, 1},-\theta_{q, 2} ; q\right\rangle_{r}}{\langle 1, h ; q\rangle_{r}} q^{r h} \epsilon_{1}^{r} \epsilon_{2}^{r} .
\end{gathered}
$$

Similarly, we obtain

$$
\begin{gathered}
\Delta_{x_{1}, x_{2} ; q}(h)=\sum_{r=0}^{\infty} \frac{\left\langle-\theta_{q, 1},-\theta_{q, 2} ; q\right\rangle_{r}}{\left\langle 1,1-h-\theta_{q, 1}-\theta_{q, 2} ; q\right\rangle_{r}} q^{r}, \\
\Delta_{x_{1}, x_{2} ; q}(h) \\
=\sum_{r=0}^{\infty} \frac{(-1)^{r}\left\langle-\theta_{q, 1},-\theta_{q, 2} ; q\right\rangle_{r}\langle h ; q\rangle_{2 r}}{\left\langle 1, h+r-1, h+\theta_{q, 1}, h+\theta_{q, 2} ; q\right\rangle_{r}} q^{\left(\begin{array}{l}
r \\
2
\end{array}\right)+r h} \epsilon_{1}^{r} \epsilon_{2}^{r}, \\
\nabla_{x_{1}, x_{2} ; q}(h) \Delta_{x_{1}, x_{2} ; q}(k) \\
=\sum_{r=0}^{\infty} \frac{\left\langle-\theta_{q, 1},-\theta_{q, 2}, k-h ; q\right\rangle_{r}\langle k ; q\rangle_{2 r}}{\left\langle 1, h, k+r-1, k+\theta_{q, 1}, k+\theta_{q, 2} ; q\right\rangle_{r}} q^{r h} \epsilon_{1}^{r} \epsilon_{2}^{r} .
\end{gathered}
$$

Definition 6. Introduce the inverse pair of operators

$$
\begin{gathered}
\widetilde{\Delta}_{x_{1} ; x_{2} x_{3} ; q}(h) \frac{1}{\langle h ; q\rangle_{m}\langle h ; q\rangle_{n+p}} x_{1}^{m} x_{2}^{n} x_{3}^{p} \\
\equiv \frac{1}{\langle h ; q\rangle_{m+n+p}} x_{1}^{m} x_{2}^{n} x_{3}^{p}, \\
\widetilde{\nabla}_{x_{1} ; x_{2} x_{3} ; q}(h) \frac{1}{\langle h ; q\rangle_{m+n+p}} x_{1}^{m} x_{2}^{n} x_{3}^{p} \\
\equiv \frac{1}{\langle h ; q\rangle_{m}\langle h ; q\rangle_{n+p}} x_{1}^{m} x_{2}^{n} x_{3}^{p} .
\end{gathered}
$$

A general operator of the type (27) is given by

$$
\widetilde{\nabla}_{x_{1} ; x_{2} \cdots x_{r} ; q}(h) \equiv \Gamma_{q}\left[\begin{array}{c}
h, h+\theta_{q, 1}+\cdots+\theta_{q, r} \\
h+\theta_{q, 1}, h+\theta_{q, 2}+\cdots+\theta_{q, r}
\end{array}\right] .
$$

We have the following summation formula for the operator $\widetilde{\nabla}_{x_{1} ; x_{2} \cdots x_{r} ; q}(h)$ :

$$
\begin{array}{r}
\widetilde{\nabla}_{x_{1} ; x_{2} \cdots x_{r} ; q}(h) \\
=\sum_{m_{2}, \ldots, m_{r}=0}^{\infty} \frac{\left\langle-\theta_{q, 1} ; q\right\rangle_{m}\left\langle-\theta_{q, 2} ; q\right\rangle_{m_{2}} \cdots\left\langle-\theta_{q, r} ; q\right\rangle_{m_{r}}}{\langle h ; q\rangle_{m}\langle 1 ; q\rangle_{m_{2}} \cdots\langle 1 ; q\rangle_{m_{r}}} \\
q^{h m} \epsilon^{m} \epsilon_{2}^{-m_{3}}\left(\epsilon_{2} \epsilon_{3}\right)^{-m_{4}} \cdots\left(\epsilon \epsilon_{1}^{-1} \epsilon_{r}^{-1}\right)^{-m_{r}},
\end{array}
$$


where

$$
m \equiv m_{2}+\cdots+m_{r}, \quad \epsilon \equiv \epsilon_{1} \cdots \epsilon_{r} .
$$

This follows from the summation formula

$$
\begin{aligned}
\Phi_{D}^{(n)} & =\left(b,-k_{1}, \ldots,-k_{n} ; c \mid q ; q^{c-b+K}, q^{c-b+K-k_{1}}, \ldots, q^{c-b+k_{n}}\right) \\
& =\frac{\langle c-b ; q\rangle_{K}}{\langle c ; q\rangle_{K}}, \quad K \equiv \sum_{i=1}^{n} k_{i},
\end{aligned}
$$

where the fourth $q$-Lauricella function is given by

$$
\begin{array}{r}
\Phi_{D}^{(n)}\left(a, b_{1}, \ldots, b_{n} ; c \mid q ; x_{1}, \ldots, x_{n}\right) \\
\equiv \sum_{\vec{m}} \frac{\langle a ; q\rangle_{m} \prod_{j=1}^{n}\left\langle b_{j} ; q\right\rangle_{m_{j}} x_{j}^{m_{j}}}{\langle c ; q\rangle_{m} \prod_{j=1}^{n}\langle 1 ; q\rangle_{m_{j}}} \\
\max \left(|x|, \ldots,\left|x_{n}\right|\right)<1 .
\end{array}
$$

\section{Computations}

The proofs of the following 15 formulas are straightforward from the definitions.

Theorem 7 (q-analogues of [1, pp. 959-960]). Consider

$$
\begin{aligned}
& H_{A}(\left(a, b_{1}, b_{2} ; c_{1}, c_{2} \mid q ; x_{1}, x_{2}, x_{3}\right) \\
&= \nabla_{x_{1}, x_{3} ; q}(a) \nabla_{x_{1}, x_{2} ; q}\left(b_{1}\right) \phi_{2}\left(a, b_{1} ; c_{1} \mid q ; x_{1}\right) \\
& \times \Phi_{1}\left(b_{2} ; b_{1}, a ; c_{2} \mid q ; x_{2}, x_{3}\right) \\
& H_{B}\left(a, b_{1}, b_{2} ; c_{1}, c, c \mid q ; x_{1}, x_{2}, x_{3}\right) \\
&=\nabla_{x_{1}, x_{3} ; q}(a) \nabla_{x_{1}, x_{2} ; q}\left(b_{1}\right) \nabla_{x_{2}, x_{3} ; q}(c) \\
& \quad \times{ }_{2} \phi_{1}\left(a, b_{1} ; c_{1} \mid q ; x_{1}\right) \\
& \quad \times \Phi_{1}\left(b_{2} ; b_{1}, a ; c \mid q ; x_{2}, x_{3}\right) \\
& H_{C}\left(a, b_{1}, b_{2} ; c \mid q ; x_{1}, x_{2}, x_{3}\right) \\
&=\nabla_{x_{1}, x_{3} ; q}(a) \nabla_{x_{1}, x_{2} ; q}\left(b_{1}\right) \widetilde{\nabla}_{x_{1} ; x_{2} x_{3} ; q}(c) \\
& \quad \times{ }_{2} \phi_{1}\left(a, b_{1} ; c \mid q ; x_{1}\right) \\
& \quad \times \Phi_{1}\left(b_{2} ; b_{1}, a ; c \mid q ; x_{2}, x_{3}\right)
\end{aligned}
$$

$$
\begin{aligned}
H_{A} & \left(a, b_{1}, b_{2} ; c_{1}, c_{2} \mid q ; x_{1}, x_{2}, x_{3}\right) \\
= & \nabla_{x_{1}, x_{3} ; q}(a) \nabla_{x_{1}, x_{2} ; q}\left(b_{1}\right) \Delta_{x_{2}, x_{3} ; q}\left(c_{2}\right) \\
& \times{ }_{2} \phi_{1}\left(a, b_{1} ; c_{1} \mid q ; x_{1}\right) \Phi_{2}\left(b_{2} ; b_{1}, a ; c_{2}, c_{2} \mid q ; x_{2}, x_{3}\right) .
\end{aligned}
$$

$H_{B}\left(a, b_{1}, b_{2} ; c_{1}, c_{2}, c_{3} \mid q ; x_{1}, x_{2}, x_{3}\right)$

$$
\begin{aligned}
= & \nabla_{x_{1}, x_{3} ; q}(a) \nabla_{x_{1}, x_{2} ; q}\left(b_{1}\right)_{2} \phi_{1}\left(a, b_{1} ; c_{1} \mid q ; x_{1}\right) \\
& \times \Phi_{2}\left(b_{2} ; b_{1}, a ; c_{2}, c_{3} \mid q ; x_{2}, x_{3}\right), \\
H_{C} & \left(a, b_{1}, b_{2} ; c \mid q ; x_{1}, x_{2}, x_{3}\right) \\
= & \nabla_{x_{1}, x_{3} ; q}(a) \nabla_{x_{1}, x_{2} ; q}\left(b_{1}\right) \Delta_{x_{2}, x_{3} ; q}(c) \widetilde{\Delta}_{x_{1} ; x_{2} x_{3} ; q}(c) \\
& \times{ }_{2} \phi_{1}\left(a, b_{1} ; c \mid q ; x_{1}\right) \Phi_{2}\left(b_{2} ; b_{1}, a ; c, c \mid q ; x_{2}, x_{3}\right),
\end{aligned}
$$

$$
\begin{aligned}
H_{A} & \left(a, b_{1}, b_{2} ; c_{1}, c_{2} \mid q ; x_{1}, x_{2}, x_{3}\right) \\
= & \nabla_{x_{1}, x_{3} ; q}(a) \nabla_{x_{1}, x_{2} ; q}\left(b_{1}\right) \nabla_{x_{2}, x_{3} ; q}\left(b_{2}\right) \\
& \quad \times{ }_{2} \phi_{1}\left(a, b_{1} ; c_{1} \mid q ; x_{1}\right) \Phi_{3}\left(b_{1}, b_{2} ; b_{2}, a ; c_{2} \mid q ; x_{2}, x_{3}\right),
\end{aligned}
$$

$$
\begin{aligned}
H_{B} & \left(a, b_{1}, b_{2} ; c_{1}, c, c \mid q ; x_{1}, x_{2}, x_{3}\right) \\
= & \nabla_{x_{1}, x_{3} ; q}(a) \nabla_{x_{1}, x_{2} ; q}\left(b_{1}\right) \nabla_{x_{2}, x_{3} ; q}\left(b_{2}\right) \nabla_{x_{2}, x_{3} ; q}(c) \\
& \quad \times{ }_{2} \phi_{1}\left(a, b_{1} ; c_{1} \mid q ; x_{1}\right) \Phi_{3}\left(b_{2}, a ; b_{1}, b_{2} ; c \mid q ; x_{2}, x_{3}\right),
\end{aligned}
$$

$$
\begin{aligned}
H_{C} & \left(a, b_{1}, b_{2} ; c \mid q ; x_{1}, x_{2}, x_{3}\right) \\
= & \nabla_{x_{1}, x_{3} ; q}(a) \nabla_{x_{1}, x_{2} ; q}\left(b_{1}\right) \nabla_{x_{2}, x_{3} ; q}\left(b_{2}\right) \widetilde{\Delta}_{x_{1} ; x_{2} x_{3} ; q}(c) \\
& \quad \times{ }_{2} \phi_{1}\left(a, b_{1} ; c \mid q ; x_{1}\right) \Phi_{3}\left(b_{2}, b_{2} ; b_{1}, a ; c \mid q ; x_{2}, x_{3}\right),
\end{aligned}
$$

$H_{A}\left(a, a, b_{2} ; c_{1}, c_{2} \mid q ; x_{1}, x_{2}, x_{3}\right)$

$$
=\nabla_{x_{1}, x_{2} ; q}(a) \nabla_{x_{1}, x_{3} ; q}(a) \Delta_{x_{2}, x_{3} ; q}(a) \Delta_{x_{2}, x_{3} ; q}\left(c_{2}\right)
$$$$
\times{ }_{2} \phi_{1}\left(a, a ; c_{1} \mid q ; x_{1}\right) \Phi_{4}\left(a ; b_{2} ; c_{2}, c_{2} q ; x_{2}, x_{3}\right),
$$

$H_{B}\left(a, a, b_{2} ; c_{1}, c_{2}, c_{3} \mid q ; x_{1}, x_{2}, x_{3}\right)$

$$
\begin{aligned}
= & \nabla_{x_{1}, x_{3} ; q}(a) \nabla_{x_{1}, x_{2} ; q}(a) \Delta_{x_{2}, x_{3} ; q}(a) \\
& \times{ }_{2} \phi_{1}\left(a, a ; c_{1} \mid q ; x_{1}\right) \Phi_{4}\left(a ; b_{2} ; c_{2}, c_{3} \mid q ; x_{2}, x_{3}\right),
\end{aligned}
$$$$
H_{C}\left(a, a, b_{2} ; c \mid q ; x_{1}, x_{2}, x_{3}\right)
$$$$
\begin{aligned}
= & \nabla_{x_{1}, x_{3} ; q}(a) \nabla_{x_{1}, x_{2} ; q}(a) \Delta_{x_{2}, x_{3} ; q}(a) \Delta_{x_{2}, x_{3} ; q}(c) \widetilde{\Delta}_{x_{1} ; x_{2} x_{3} ; q}(c) \\
& \times{ }_{2} \phi_{1}\left(a, a ; c \mid q ; x_{1}\right) \Phi_{4}\left(a ; b b_{2} ; c, c \mid q ; x_{2}, x_{3}\right)
\end{aligned}
$$ 


$$
\begin{aligned}
H_{A} & \left(a, b_{1}, b_{2} ; c_{1}, c_{2} \mid q ; x_{1}, x_{2}, x_{3}\right) \\
= & \nabla_{x_{1}, x_{3} ; q}(a) \nabla_{x_{1}, x_{2} ; q}\left(b_{1}\right) \nabla_{x_{2} x_{3} ; q}\left(b_{2}\right) \Delta_{x_{2}, x_{3} ; q}\left(c_{2}\right) \\
& \times{ }_{2} \phi_{1}\left(a, b_{1} ; c_{1} \mid q ; x_{1}\right){ }_{2} \phi_{1}\left(b_{1}, b_{2} ; c_{2} \mid q ; x_{2}\right) \\
& \times{ }_{2} \phi_{1}\left(a, b_{2} ; c_{2} \mid q ; x_{3}\right) \\
H_{B} & \left(a, b_{1}, b_{2} ; c_{1}, c_{2}, c_{3} \mid q ; x_{1}, x_{2}, x_{3}\right) \\
= & \nabla_{x_{1}, x_{3} ; q}(a) \nabla_{x_{1}, x_{2} ; q}\left(b_{1}\right) \nabla_{x_{2}, x_{3} ; q}\left(b_{2}\right) \\
& \times{ }_{2} \phi_{1}\left(a, b_{1} ; c_{1} \mid q ; x_{1}\right){ }_{2} \phi_{1}\left(b_{1}, b_{2} ; c_{2} \mid q ; x_{2}\right) \\
& \times{ }_{2} \phi_{1}\left(a, b_{2} ; c_{3} \mid q ; x_{3}\right) \\
H_{C} & \left(a, b_{1}, b_{2} ; c \mid q ; x_{1}, x_{2}, x_{3}\right) \\
= & \nabla_{x_{1}, x_{3} ; q}(a) \nabla_{x_{1}, x_{2} ; q}\left(b_{1}\right) \nabla_{x_{2}, x_{3} ; q}\left(b_{2}\right) \Delta_{x_{2}, x_{3} ; q}(c) \\
& \times \widetilde{\Delta}_{x_{1} ; x_{2} x_{3} ; q}(c){ }_{2} \phi_{1}\left(a, b_{1} ; c \mid q ; x_{1}\right)_{2} \phi_{1}\left(b_{1}, b_{2} ; c \mid q ; x_{2}\right) \\
& \times{ }_{2} \phi_{1}\left(a, b_{2} ; c \mid q ; x_{3}\right) .
\end{aligned}
$$

Remark 8 . We note that there are $3 \times 5=15$ equations and $H_{A}, H_{B}$, and $H_{C}$ always follow after each other five times. The operator $\widetilde{\nabla}_{x_{1} ; x_{2} x_{3} ; q}$ always follows after $H_{C}$. The slightly more involved proofs of formulas (42) to (44) are somehow connected. The formulas are symmetrically ordered (in triples to the far right) according to the scheme $\Phi_{1}, \Phi_{2}, \Phi_{3}, \Phi_{4},{ }_{2} \phi_{1}$. We have corrected a slight error in formula [1, p. 959 (3.6)].

We will now present $10 q$-analogues of the improved versions of [1, pp. 960-962]. The proofs are quite similar; in each case, we obtain a 2-, 3-, or 4-sum (denoted by the letters $i, j, k, l)$, followed by a three-sum mnp, which denotes a $q$-hypergeometric function times a $q$-Appell function. The most difficult part in each proof is the variable transformation which takes place at the step denoted by. If we denote the variables at this stage by $i, j, k, l, r, m, n, p$ (the old summation indices) and $i^{\prime}, j^{\prime}, k^{\prime}, l^{\prime}, r^{\prime}, m^{\prime}, n^{\prime}, p^{\prime}$ (the new summation indices), we have to find a symmetric variable transformation with nonzero determinant. The two first transformations take the forms:

$$
\begin{array}{r}
m=i^{\prime}+j^{\prime}+m^{\prime}, \quad n=j^{\prime}+n^{\prime}, \quad \begin{array}{c}
p=j^{\prime}+p^{\prime}, \\
i=i^{\prime}, j=j^{\prime}, \\
m=i^{\prime}+j^{\prime}+m^{\prime}, \quad n=j^{\prime}+k^{\prime}+n^{\prime}, \quad p=i^{\prime}+k^{\prime}+p^{\prime}, \\
i=i^{\prime}, \quad j=j^{\prime}, k=k^{\prime} .
\end{array}
\end{array}
$$

Throughout, we use the following abbreviations:

$$
\begin{aligned}
& X^{M} \equiv x_{1}^{m} x_{2}^{n} x_{3}^{p}, \quad C_{2} \equiv c_{2}+k, \\
& H_{A}\left(a, b_{1}, b_{2} ; c_{1}, c_{2} \mid q ; x_{1}, x_{2}, x_{3}\right) \\
& =\sum_{i j} \frac{\left\langle 1, a, b_{1}, b_{2} ; q\right\rangle_{i+j}}{\langle 1.1 ; q\rangle_{i}\left\langle c_{1}, c_{2} ; q\right\rangle_{i+j}\langle 1,1 ; q\rangle_{j}} \\
& \times \frac{x_{1}^{i+j} x_{2}^{j} x_{3}^{i} q^{i(a+i-1)+j\left(b_{1}+j-1\right)}}{\left\langle 1, b_{1} ; q\right\rangle_{i+k}\langle 1 ; q\rangle_{i-k}\langle 1 ; q\rangle_{n}\langle 1 ; q\rangle_{p}} \\
& \times{ }_{4} \phi_{3}\left[\begin{array}{c}
a+i+j, b_{1}+i+j, 1+i+j, 1 \\
1+i, 1+j, c_{1}+i+j
\end{array}\right. \\
& \times \Phi_{1}\left(b_{2}+i+j ; b_{1}+j, a+i ; c_{1}+i+j \mid q ; x_{2}, x_{3}\right) .
\end{aligned}
$$

Proof. Use formula (33). The computation goes as follows:

$$
\begin{aligned}
H_{A}\left(a, b_{1}, b_{2} ; c_{1}, c_{2} \mid q ; x_{1}, x_{2}, x_{3}\right) & \\
=\sum_{i j m n p} & \frac{\langle-m,-p ; q\rangle_{i} q^{i(a+m+p)}\langle-m,-n ; q\rangle_{j} q^{j\left(b_{1}+m+n\right)}}{\langle 1, a ; q\rangle_{i}\left\langle 1, b_{1} ; q\right\rangle_{j}} \\
\times & X^{M} \frac{\left\langle a, b_{1} ; q\right\rangle_{m}\left\langle b_{2} ; q\right\rangle_{n+p}\left\langle b_{1} ; q\right\rangle_{n}\langle a ; q\rangle_{p}}{\left\langle 1, c_{1} ; q\right\rangle_{m}\left\langle c_{2} ; q\right\rangle_{n+p}\langle 1 ; q\rangle_{n}\langle 1 ; q\rangle_{p}} \\
=\sum_{i j m n p} & \frac{q^{i(a+i-1)+j\left(b_{1}+j-1\right)}}{\langle 1 ; q\rangle_{m-i}\langle 1, a ; q\rangle_{i}\langle 1 ; q\rangle_{m-j}\langle 1 ; q\rangle_{p-i}\left\langle 1, b_{1} ; q\right\rangle_{j}} \\
\times \sum_{i j m n p} & \left(\langle 1 , a , b _ { 1 } ; q \rangle _ { m + i + j } \left\langle\frac{\left\langle 1, a, b_{1} ; q\right\rangle_{m}\left\langle b_{2} ; q\right\rangle_{n+p}\left\langle b_{1} ; q\right\rangle_{n}\langle a ; q\rangle_{p}}{\langle 1 ; q\rangle_{n-j}\left\langle c_{1} ; q\right\rangle_{m}\left\langle c_{2} ; q\right\rangle_{n+p}\left\langle b_{1} ; q\right\rangle_{j+n}}\right.\right. \\
\times & \left.\times\left\langle b_{2} ; q\right\rangle_{i+j+n+p} x_{1}^{m+i+j} x_{2}^{j+n} x_{3}^{i+p}\right) \\
\times & \left(\langle 1 ; q\rangle_{m+i}\langle 1 ; q\rangle_{p}\langle 1, a ; q\rangle_{i}\left\langle 1, b_{1} ; q\right\rangle_{j}\langle 1 ; q\rangle_{m+j}\right)^{-1} \\
\times & \frac{q^{i(a+i-1)+j\left(b_{1}+j-1\right)}}{\langle 1 ; q\rangle_{n}\left\langle c_{2} ; q\right\rangle_{i+j+n+p}\left\langle c_{1} ; q\right\rangle_{m+i+j}}=\text { RHS. }
\end{aligned}
$$

The limit case $x_{3}=0$ in (50) leads to (75). Consider

$$
\begin{aligned}
& H_{B}\left(a, b_{1}, b_{2} ; c_{1}, c, c \mid q ; x_{1}, x_{2}, x_{3}\right) \\
& =\sum_{i j k}\left\langle 1, a, b_{1} ; q\right\rangle_{i+j}\left\langle b_{2} ; q\right\rangle_{i+j+2 k} Q E\left(j\left(b_{1}+j-1\right)\right) \\
& \quad \times \frac{q^{i(a+i-1)+k(c+k-1)} x_{1}^{i+j} x_{2}^{j+k} x_{3}^{i+k}\langle 1, a ; q\rangle_{i+k}\left\langle 1, b_{1} ; q\right\rangle_{j+k}}{\left\langle 1,1,1, b_{1} ; q\right\rangle_{j}\langle 1,1,1, a ; q\rangle_{i}\langle 1,1,1, c ; q\rangle_{k}\left\langle c_{1} ; q\right\rangle_{i+j}\langle c ; q\rangle_{i+j+2 k}} \\
& \quad \times{ }_{4} \phi_{3}\left[\begin{array}{c}
1, a+i+j, b_{1}+i+j, 1+i+j \\
1+j, c_{1}+i+j, 1+i
\end{array}\right) \\
& \quad \times \Phi_{1: 2}^{1: 3}\left[\begin{array}{c}
b_{2}+i+j+2 k: 1, b_{1}+j+k, 1+j+k ; 1, a+i+k, 1+i+k \\
c+i+j+2 k: 1+k, 1+j ; 1+k, 1+i
\end{array}\right]
\end{aligned}
$$


Proof. Use formula (34). The very symmetric computation goes as follows:

$$
\begin{aligned}
& H_{B}\left(a, b_{1}, b_{2} ; c_{1}, c, c \mid q ; x_{1}, x_{2}, x_{3}\right) \\
&=\sum_{i j k m n p} \frac{\langle-m,-p ; q\rangle_{i} q^{i(a+m+p)}\langle-m,-n ; q\rangle_{j} q^{j\left(b_{1}+m+n\right)}\langle-n,-p ; q\rangle_{k} q^{k(c+n+p)}}{\langle 1, a ; q\rangle_{i}\left\langle 1, b_{1} ; q\right\rangle_{j}\langle 1, c ; q\rangle_{k}} \\
& \quad \times X^{M} \frac{\left\langle a, b_{1} ; q\right\rangle_{m}\left\langle b_{2} ; q\right\rangle_{n+p}\left\langle b_{1} ; q\right\rangle_{n}\langle a ; q\rangle_{p}}{\left\langle 1, c_{1} ; q\right\rangle_{m}\langle c ; q\rangle_{n+p}\langle 1 ; q\rangle_{n}\langle 1 ; q\rangle_{p}} \\
&=\sum_{i j k m n p} \frac{q 1 ; q\rangle_{m-i}\langle 1, a ; q\rangle_{i}\langle 1 ; q\rangle_{m-j}\langle 1 ; q\rangle_{p-i}\langle 1 ; q\rangle_{p-k}\left\langle 1, b_{1} ; q\right\rangle_{j}\langle 1, c ; q\rangle_{k}}{\left\langle(a+i-1)+j\left(b_{1}+j-1\right)+k(c+k-1)\right.} \\
& \quad \times X^{M} \frac{\left\langle 1, a, b_{1} ; q\right\rangle_{m}\left\langle b_{2} ; q\right\rangle_{n+p}\left\langle 1, b_{1} ; q\right\rangle_{n}\langle 1, a ; q\rangle_{p}}{\langle 1 ; q\rangle_{n-j}\langle 1 ; q\rangle_{n-k}\left\langle c_{1} ; q\right\rangle_{m}\langle c ; q\rangle_{n+p}} \\
& \sum_{i j k m n p} \frac{\left\langle 1, a, b_{1} ; q\right\rangle_{i+j+m}\langle 1, a ; q\rangle_{i+k+p}\left\langle 1, b_{1} ; q\right\rangle_{j+k+n}\left\langle b_{2} ; q\right\rangle_{i+j+2 k+n+p} x_{1}^{i+j+m}}{\langle 1 ; q\rangle_{m+j}\langle 1 ; q\rangle_{m+i}\langle 1 ; q\rangle_{k+p}\langle 1 ; q\rangle_{i+p}\langle 1, a ; q\rangle_{i}\left\langle 1, b_{1} ; q\right\rangle_{j}\langle 1, c ; q\rangle_{k}\langle 1 ; q\rangle_{j+n}} \\
& \times \frac{x_{2}^{j+n+k} x_{3}^{i+k+p} q^{i(a+i-1)+j\left(b_{1}+j-1\right)+k(c+k-1)}}{\langle 1 ; q\rangle_{k+n}\langle c ; q\rangle_{i+j+2 k+n+p}\left\langle c_{1} ; q\right\rangle_{i+j+m}}=\mathrm{RHS} .
\end{aligned}
$$

The limit case $x_{1}=0$ in (52) leads to (76). Consider

$$
\begin{aligned}
& H_{A}\left(a, b_{1}, b_{2} ; c_{1}, c_{2} \mid q ; x_{1}, x_{2}, x_{3}\right) \\
& =\sum_{i j k} \frac{q^{i(a+i-1)+j\left(b_{1}+j-1\right)}\left\langle 1, a, b_{1} ; q\right\rangle_{i+j} x_{1}^{i+j} x_{2}^{k+j} x_{3}^{i+k} q^{(3 / 2)\left(k^{2}-k\right)+k c_{2}}}{\left\langle C_{2} ; q\right\rangle_{j+k}\left\langle C_{2} ; q\right\rangle_{i+k}} \\
& \quad \times \frac{\left\langle 1, b_{1} ; q\right\rangle_{k+j}\left\langle b_{2} ; q\right\rangle_{i+j+2 k}(-1)^{k}\left\langle c_{2} ; q\right\rangle_{2 k}\langle 1, a ; q\rangle_{i+k}\langle 1+i+k, a+i+k ; q\rangle_{p}}{\langle 1,1,1, a ; q\rangle_{i}\left\langle 1,1,1, b_{1} ; q\right\rangle_{j}\left\langle c_{1} ; q\right\rangle_{i+j}\left\langle 1,1,1, C_{2}-1, c_{2}, c_{2} ; q\right\rangle_{k}} \\
& \left.\quad \times{ }_{4} \phi_{3}\left[\begin{array}{c}
1,1+i+j, a+i+j, b_{1}+i+j \\
1+i, 1+j, c_{1}+i+j
\end{array}\right] q ; x_{1}\right] \\
& \quad \times \Phi_{1: 3}^{1: 4}\left[\begin{array}{c}
b_{2}+i+j+2 k: 1,1+k+j, b_{1}+k+j, \infty ; 1,1+i+k, a+i+k, \infty \\
\infty: c_{2}+j+2 k, 1+k, 1+j ; c_{2}+i+2 k, 1+i, 1+k
\end{array}\right] .
\end{aligned}
$$


Proof. Use formula (36). The computation goes as follows:

$$
\begin{aligned}
& H_{A}\left(a, b_{1}, b_{2} ; c_{1}, c_{2} \mid q ; x_{1}, x_{2}, x_{3}\right) \\
& =\sum_{i j k m n p} \frac{\langle-m,-p ; q\rangle_{i} q^{i(a+m+p)}\langle-m,-n ; q\rangle_{j} q^{j\left(b_{1}+m+n\right)}(-1)^{k}\langle-n,-p ; q\rangle_{k}\left\langle c_{2} ; q\right\rangle_{2 k}}{\langle 1, a ; q\rangle_{i}\left\langle 1, b_{1} ; q\right\rangle_{j}\left\langle 1, C_{2}-1, c_{2}+n, c_{2}+p ; q\right\rangle_{k}} \\
& \times X^{M} \frac{\left\langle a, b_{1} ; q\right\rangle_{m}\left\langle b_{2} ; q\right\rangle_{n+p}\left\langle b_{1} ; q\right\rangle_{n}\langle a ; q\rangle_{p}}{\left\langle 1, c_{1} ; q\right\rangle_{m}\left\langle 1, c_{2} ; q\right\rangle_{n}\left\langle 1, c_{2} ; q\right\rangle_{p}} q^{\left(\begin{array}{c}
k \\
2
\end{array}\right)+k\left(c_{2}+n+p\right)} \\
& =\sum_{i j k m n p} \frac{\left\langle 1, a, b_{1} ; q\right\rangle_{m} q^{i(a+i-1)+j\left(b_{1}+j-1\right)}\langle 1, a ; q\rangle_{p}(-1)^{k}\left\langle c_{2} ; q\right\rangle_{2 k}}{\langle 1 ; q\rangle_{m-i}\langle 1 ; q\rangle_{m-j}\langle 1, a ; q\rangle_{i}\left\langle 1, b_{1} ; q\right\rangle_{j}\langle 1 ; q\rangle_{p-i}} \\
& \times \frac{X^{M}\left\langle b_{2} ; q\right\rangle_{n+p}\left\langle 1, b_{1} ; q\right\rangle_{n} q^{(3 / 2)\left(k^{2}-k\right)+k c_{2}}}{\langle 1 ; q\rangle_{n-j}\langle 1 ; q\rangle_{n-k}\left\langle c_{1} ; q\right\rangle_{m}\left\langle C_{2} ; q\right\rangle_{n}\left\langle C_{2} ; q\right\rangle_{p}\langle 1 ; q\rangle_{p-k}\left\langle 1, C_{2}-1, c_{2}, c_{2} ; q\right\rangle_{k}} \\
& \stackrel{\star}{=} \sum_{i j k m n p} \frac{q^{i(a+i-1)+j\left(b_{1}+j-1\right)}\left\langle 1, a, b_{1} ; q\right\rangle_{m+i+j} x_{1}^{m+i+j} x_{2}^{n+k+j} x_{3}^{p+i+k} q^{(3 / 2)\left(k^{2}-k\right)+k c_{2}}}{\left\langle 1, C_{2}-1, c_{2}, c_{2} ; q\right\rangle_{k}\left\langle C_{2} ; q\right\rangle_{n+j+k}\left\langle C_{2} ; q\right\rangle_{p+i+k}\langle 1, a ; q\rangle_{i}\left\langle 1, b_{1} ; q\right\rangle_{j}} \\
& \times \frac{(-1)^{k}\left\langle c_{2} ; q\right\rangle_{2 k}\left\langle b_{2} ; q\right\rangle_{n+p+i+j+2 k}\langle 1, a ; q\rangle_{p+i+k}\left\langle 1, b_{1} ; q\right\rangle_{n+k+j}}{\langle 1 ; q\rangle_{m+j}\langle 1 ; q\rangle_{m+i}\langle 1 ; q\rangle_{p+k}\langle 1 ; q\rangle_{p+i}\langle 1 ; q\rangle_{n+k}\langle 1 ; q\rangle_{n+j}\left\langle c_{1} ; q\right\rangle_{m+i+j}}=\text { RHS, } \\
& H_{B}\left(a, b_{1}, b_{2} ; c_{1}, c_{2}, c_{3} \mid q ; x_{1}, x_{2}, x_{3}\right) \\
& =\sum_{i j} \frac{\left\langle 1, a, b_{1}, b_{2} ; q\right\rangle_{i+j}}{\left\langle 1,1, c_{3} ; q\right\rangle_{i}\left\langle c_{1} ; q\right\rangle_{i+j}\left\langle 1,1, c_{2} ; q\right\rangle_{j}} \\
& \times x_{1}^{i+j} x_{2}^{j} x_{3}^{i} q^{i(a+i-1)+j\left(b_{1}+j-1\right)}{ }_{4} \phi_{3}\left[\begin{array}{c}
a+i+j, b_{1}+i+j, 1+i+j, 1 \\
1+i, 1+j, c_{1}+i+j
\end{array} \mid q ; x_{1}\right] \\
& \times \Phi_{1: 1}^{1: 2}\left[\begin{array}{cc}
b_{2}+i+j: b_{1}+j, \infty ; a+i, \infty & \mid q ; x_{2}, x_{3} \\
\infty: c_{2}+j ; c_{3}+i & .
\end{array}\right.
\end{aligned}
$$

Proof. Use formula (37). The computation goes as follows:

$$
\begin{aligned}
H_{B} & \left(a, b_{1}, b_{2} ; c_{1}, c_{2}, c_{3} \mid q ; x_{1}, x_{2}, x_{3}\right) \\
& =\sum_{i j m n p} \frac{\langle-m,-p ; q\rangle_{i} q^{i(a+m+p)}\langle-m,-n ; q\rangle_{j} q^{j\left(b_{1}+m+n\right)}}{\langle 1, a ; q\rangle_{i}\left\langle 1, b_{1} ; q\right\rangle_{j}} X^{M} \frac{\left\langle a, b_{1} ; q\right\rangle_{m}\left\langle b_{2} ; q\right\rangle_{n+p}\left\langle b_{1} ; q\right\rangle_{n}\langle a ; q\rangle_{p}}{\left\langle 1, c_{1} ; q\right\rangle_{m}\left\langle 1, c_{2} ; q\right\rangle_{n}\left\langle 1, c_{3} ; q\right\rangle_{p}} \\
& =\sum_{i j m n p} \frac{\langle 1 ; q\rangle_{m-i}\langle 1, a ; q\rangle_{i}\langle 1 ; q\rangle_{m-j}\langle 1 ; q\rangle_{p-i}\left\langle 1, b_{1} ; q\right\rangle_{j}\langle 1 ; q\rangle_{n-j}\left\langle c_{1} ; q\right\rangle_{m}\left\langle c_{2} ; q\right\rangle_{n}\left\langle c_{3} ; q\right\rangle_{p}}{\langle 1 ; q\rangle_{n+p}\left\langle b_{1} ; q\right\rangle_{n}\langle a ; q\rangle_{p} q^{i(a+i-1)+j\left(b_{1}+j-1\right)} X^{M}} \\
& \stackrel{\star}{=} \sum_{i j m n p} \frac{\left\langle 1, a, b_{1} ; q\right\rangle_{m+i+j}\langle a ; q\rangle_{i+p}\left\langle b_{1} ; q\right\rangle_{j+n}\left\langle b_{2} ; q\right\rangle_{i+j+n+p} x_{1}^{m+i+j} x_{2}^{j+n} x_{3}^{i+p}}{\langle 1 ; q\rangle_{m+i}\langle 1 ; q\rangle_{m+j}\langle 1 ; q\rangle_{p}\langle 1, a ; q\rangle_{i}\left\langle 1, b_{1} ; q\right\rangle_{j}\langle 1 ; q\rangle_{n}} \frac{q^{i(a+i-1)+j\left(b_{1}+j-1\right)}}{\left\langle c_{1} ; q\right\rangle_{m+i+j}\left\langle c_{2} ; q\right\rangle_{n+j}\left\langle c_{3} ; q\right\rangle_{p+i}}=\text { RHS. }
\end{aligned}
$$

The limit case $x_{3}=0$ in (56) leads to (75). Consider 


$$
\begin{aligned}
H_{A}\left(a, b_{1}, b_{2} ; c_{1}, c_{2} \mid q ; x_{1}, x_{2}, x_{3}\right) \\
=\sum_{i j k} \frac{\left\langle 1, a, b_{1} ; q\right\rangle_{i+j}}{\left\langle c_{1} ; q\right\rangle_{i+j}} x_{1}^{i+j} x_{2}^{j+k} x_{3}^{i+k} \\
\quad \times \frac{q^{i(a+i-1)+k\left(b_{2}+k-1\right)+j\left(b_{1}+j-1\right)}\left\langle 1, a, b_{2} ; q\right\rangle_{i+k}\left\langle 1, b_{1}, b_{2} ; q\right\rangle_{j+k}}{\left\langle 1,1,1, b_{1} ; q\right\rangle_{j}\langle 1,1,1, a ; q\rangle_{i}\left\langle 1,1,1, b_{2} ; q\right\rangle_{k}\left\langle c_{2} ; q\right\rangle_{i+j+2 k}} \\
\quad \times{ }_{4} \phi_{3}\left[\begin{array}{c}
1, a+i+j, b_{1}+i+j, 1+i+j \\
1+j, c_{1}+i+j, 1+i
\end{array}\right] \\
\quad \times \Phi_{1: 3}^{1: 4}\left[\begin{array}{c}
\left.\infty: 1, x_{1}+j+k, 1+j+k, b_{2}+j+k ; 1, a+i+k, 1+i+k, b_{2}+i+k \quad \mid q ; x_{2}, x_{3}\right] . \\
c_{2}+i+j+2 k: \infty, 1+k, 1+j ; \infty, 1+i, 1+k
\end{array}\right.
\end{aligned}
$$

Proof. Use formula (39). The computation goes as follows:

$$
\begin{aligned}
& H_{A}\left(a, b_{1}, b_{2} ; c_{1}, c_{2} \mid q ; x_{1}, x_{2}, x_{3}\right) \\
&=\sum_{i j k m n p} \frac{\langle-m,-p ; q\rangle_{i} q^{i(a+m+p)}\langle-m,-n ; q\rangle_{j} q^{j\left(b_{1}+m+n\right)}\langle-n,-p ; q\rangle_{k} q^{k\left(b_{2}+n+p\right)}}{\langle 1, a ; q\rangle_{i}\left\langle 1, b_{1} ; q\right\rangle_{j}\left\langle 1, b_{2} ; q\right\rangle_{k}} \\
& \quad \times X^{M} \frac{\left\langle a, b_{1} ; q\right\rangle_{m}\left\langle b_{1}, b_{2} ; q\right\rangle_{n}\left\langle a, b_{2} ; q\right\rangle_{p}}{\left\langle 1, c_{1} ; q\right\rangle_{m}\left\langle c_{2} ; q\right\rangle_{n+p}\langle 1 ; q\rangle_{n}\langle 1 ; q\rangle_{p}} \\
&=\sum_{i j k m n p} \frac{q^{i(a+i-1)+j\left(b_{1}+j-1\right)+k\left(b_{2}+k-1\right)}}{\langle 1 ; q\rangle_{m-i}\langle 1, a ; q\rangle_{i}\langle 1 ; q\rangle_{m-j}\langle 1 ; q\rangle_{p-i}\langle 1 ; q\rangle_{p-k}\left\langle 1, b_{1} ; q\right\rangle_{j}\left\langle 1, b_{2} ; q\right\rangle_{k}} \\
& \quad \times X^{M} \frac{\left\langle 1, a, b_{1} ; q\right\rangle_{m}\left\langle 1, b_{1}, b_{2} ; q\right\rangle_{n}\left\langle 1, a, b_{2} ; q\right\rangle_{p}}{\langle 1 ; q\rangle_{n-j}\langle 1 ; q\rangle_{n-k}\left\langle c_{1} ; q\right\rangle_{m}\left\langle c_{2} ; q\right\rangle_{n+p}} \\
& \sum_{i j k m n p} \frac{\langle 1 ; q\rangle_{m+j}\langle 1 ; q\rangle_{m+i}\langle 1 ; q\rangle_{k+p}\langle 1 ; q\rangle_{i+p}\langle 1, a ; q\rangle_{i}\left\langle 1, b_{1} ; q\right\rangle_{j}\left\langle 1, b_{2} ; q\right\rangle_{k}}{\left\langle 1, b_{1} ; q\right\rangle_{i+j+m}\left\langle 1, a, b_{2} ; q\right\rangle_{i+k+p}\left\langle 1, b_{1}, b_{2} ; q\right\rangle_{j+k+n} x_{1}^{i+j+m}} \\
& \quad \times \frac{x_{2}^{j+n+k} x_{3}^{i+k+p} q^{i(a+i-1)+j\left(b_{1}+j-1\right)+k\left(b_{2}+k-1\right)}}{\langle 1 ; q\rangle_{j+n}\langle 1 ; q\rangle_{k+n}\left\langle c_{2} ; q\right\rangle_{i+j+2 k+n+p}\left\langle c_{1} ; q\right\rangle_{i+j+m}}=\mathrm{RHS.}
\end{aligned}
$$

Put

$$
\begin{gathered}
\alpha \equiv\left(1,1+j+k+l, 1+j+k+l, b_{1}+j+k+l, b_{2}+j+k+l\right) \\
\left(1,1+i+k+l, 1+i+k+l, a+i+k+l, b_{2}+i+k+l\right) \\
\beta \equiv(1+j+k, 1+k+l, 1+j+l, \infty) \\
(1+k+l, 1+i+l, 1+i+k, \infty) .
\end{gathered}
$$


Then,

$$
\begin{aligned}
& H_{B}\left(a, b_{1}, b_{2} ; c_{1}, c, c \mid q ; x_{1}, x_{2}, x_{3}\right) \\
& =\sum_{i j k l} \frac{\left\langle 1, a, b_{1} ; q\right\rangle_{i+j} q^{j\left(b_{1}+j-1\right)+l(c+l-1)}}{\langle 1,1 ; q\rangle_{k+l}\langle 1 ; q\rangle_{i+l}\langle 1 ; q\rangle_{j+l}\langle 1 ; q\rangle_{j+k}\langle 1 ; q\rangle_{i+k}\langle 1, c ; q\rangle_{l}} \\
& \quad \times \frac{q^{i(a+i-1)+k\left(b_{2}+k-1\right)} x_{1}^{i+j} x_{2}^{j+k+l} x_{3}^{i+k+l}\left\langle 1,1, a, b_{2} ; q\right\rangle_{i+k+l}\left\langle 1,1, b_{1}, b_{2} ; q\right\rangle_{j+k+l}}{\left\langle 1,1, b_{1} ; q\right\rangle_{j}\langle 1,1, a ; q\rangle_{i}\left\langle 1, b_{2} ; q\right\rangle_{k}\left\langle c_{1} ; q\right\rangle_{i+j}\langle c ; q\rangle_{i+j+2 k+2 l}} \\
& \quad \times{ }_{4} \phi_{3}\left[\begin{array}{c}
1, a+i+j, b_{1}+i+j, 1+i+j \\
1+j, c_{1}+i+j, 1+i
\end{array} \mid q ; x_{1}\right] \\
& \quad \times \Phi_{1: 4}^{1: 5}\left[\begin{array}{c}
c+i+j+2 k+2 l: \beta \mid q ; x_{2}, x_{3} \\
c+i+j
\end{array}\right] .
\end{aligned}
$$

Proof. Use formula (40). The computation goes as follows:

$$
\begin{aligned}
& H_{B}\left(a, b_{1}, b_{2} ; c_{1}, c, c \mid q ; x_{1}, x_{2}, x_{3}\right) \\
& =\sum_{i j k l m n p} \frac{\langle-m,-p ; q\rangle_{i} q^{i(a+m+p)}\langle-m,-n ; q\rangle_{j} q^{j\left(b_{1}+m+n\right)}\langle-n,-p ; q\rangle_{k}}{\langle 1, a ; q\rangle_{i}\left\langle 1, b_{1} ; q\right\rangle_{j}\left\langle 1, b_{2} ; q\right\rangle_{k}\langle 1, c ; q\rangle_{l}} \\
& \quad \times X^{M} \frac{\left\langle a, b_{1} ; q\right\rangle_{m}\left\langle b_{1}, b_{2} ; q\right\rangle_{n}\left\langle a, b_{2} ; q\right\rangle_{p}\langle-n,-p ; q\rangle_{l} q^{k\left(b_{2}+n+p\right)+l(c+n+p)}}{\left\langle 1, c_{1} ; q\right\rangle_{m}\langle c ; q\rangle_{n+p}\langle 1 ; q\rangle_{n}\langle 1 ; q\rangle_{p}} \\
& =\sum_{i j k l m n p} \frac{\left\langle 1, a, b_{1} ; q\right\rangle_{m} q^{i(a+i-1)+j\left(b_{1}+j-1\right)+k\left(b_{2}+k-1\right)+l(c+l-1)}}{\langle 1 ; q\rangle_{m-i}\langle 1, a ; q\rangle_{i}\langle 1 ; q\rangle_{m-j}\langle 1 ; q\rangle_{p-i}\langle 1 ; q\rangle_{p-k}\left\langle 1, b_{1} ; q\right\rangle_{j}\left\langle 1, b_{2} ; q\right\rangle_{k}} \\
& \quad \times X^{M} \frac{\left\langle 1,1, b_{1}, b_{2} ; q\right\rangle_{n}\left\langle 1,1, a, b_{2} ; q\right\rangle_{p}}{\langle 1 ; q\rangle_{n-j}\langle 1 ; q\rangle_{n-k}\langle 1 ; q\rangle_{n-l}\langle 1 ; q\rangle_{p-l}\left\langle c_{1} ; q\right\rangle_{m}\langle c ; q\rangle_{n+p}\langle 1, c ; q\rangle_{l}} \\
& \quad \sum_{i j k l m n p} \frac{\left\langle 1, a, b_{1} ; q\right\rangle_{i+j+m}\left\langle 1,1, a, b_{2} ; q\right\rangle_{i+k+l+p}\left\langle 1,1, b_{1}, b_{2} ; q\right\rangle_{j+k+l+n}}{\langle 1 ; q\rangle_{m+j}\langle 1 ; q\rangle_{m+i}\langle 1 ; q\rangle_{k+l+p}\langle 1 ; q\rangle_{i+l+p}\langle 1 ; q\rangle_{i+k+p}\langle 1, a ; q\rangle_{i}\left\langle 1, b_{1} ; q\right\rangle_{j}\left\langle 1, b_{2} ; q\right\rangle_{k}} \\
& \times \frac{x_{1}^{i+j+m} x_{2}^{j+k+l+n} x_{3}^{i+k+l+p} q^{i(a+i-1)+j\left(b_{1}+j-1\right)+k\left(b_{2}+k-1\right)+l(c+l-1)}}{\langle 1 ; q\rangle_{j+k+n}\langle 1 ; q\rangle_{k+l+n}\left\langle c_{1} ; q\right\rangle_{i+j+m}\langle c ; q\rangle_{i+j+2 k+2 l+n+p}\langle 1, c ; q\rangle_{l}\langle 1 ; q\rangle_{j+l+n}}=\text { RHS. }
\end{aligned}
$$

Put

$$
\begin{aligned}
\alpha \equiv & (1,1+j+k+l, 1+j+k+l, a+j+k+l, \infty, \infty) ; \\
& (1,1+i+k+l, 1+i+k+l, a+i+k+l, \infty, \infty), \\
\beta \equiv & \left(1+l+k, 1+j+l, 1+j+k, C_{2}\right. \\
& +j+k+l, a+j+k+2 l) ; \\
& \left(1+k+l, 1+i+k, 1+i+l, C_{2}\right. \\
& +i+k+l, a+i+k+2 l) .
\end{aligned}
$$


Then,

$$
\begin{aligned}
& H_{A}\left(a, a, b_{2} ; c_{1}, c_{2} \mid q ; x_{1}, x_{2}, x_{3}\right) \\
& =\sum_{i j k l} \frac{q^{i(a+i-1)+j(a+j-1)+(3 / 2)\left(k^{2}-k\right)+k c_{2}+(3 / 2)\left(l^{2}-l\right)+l a}\langle 1, a, a ; q\rangle_{i+j} x_{1}^{i+j} x_{2}^{k+j+l} x_{3}^{i+k+l}}{\left\langle C_{2} ; q\right\rangle_{j+k+l}\left\langle C_{2} ; q\right\rangle_{i+k+l}\langle 1,1, a ; q\rangle_{i}\langle 1,1, a ; q\rangle_{j}\langle 1 ; q\rangle_{j+l}\langle 1 ; q\rangle_{j+k}} \\
& \quad \times \frac{\left\langle a, b_{2} ; q\right\rangle_{i+j+2 k+2 l}(-1)^{k+l}\left\langle c_{2} ; q\right\rangle_{2 k}\langle a ; q\rangle_{2 l}}{\langle 1,1 ; q\rangle_{k+l}\langle 1 ; q\rangle_{i+k}\langle 1 ; q\rangle_{i+l}\left\langle c_{1} ; q\right\rangle_{i+j}\left\langle 1, C_{2}-1, c_{2}, c_{2} ; q\right\rangle_{k}\langle 1, a+l-1, a, a ; q\rangle_{l}} \\
& \quad \times \frac{\langle 1,1, a ; q\rangle_{j+k+l}\langle 1,1, a ; q\rangle_{i+k+l}}{\langle a+l ; q\rangle_{j+k+l}\langle a+l ; q\rangle_{i+k+l}}{ }_{4} \phi_{3}\left[\begin{array}{c}
1,1+i+j, a+i+j, a+i+j \\
1+i, 1+j, c_{1}+i+j
\end{array}\right] \\
& \quad \times \Phi_{2: 5}^{2: 6}\left[a+i+j+2 k+2 l, b_{2}+i+j+2 k+2 l: \alpha \quad \mid q ; x_{2}, x_{3}\right] .
\end{aligned}
$$

Proof. Use formula (42). The computation goes as follows:

$$
\begin{aligned}
& H_{A}\left(a, a, b_{2} ; c_{1}, c_{2} \mid q ; x_{1}, x_{2}, x_{3}\right) \\
& =\sum_{i j k l m n p} \frac{X^{M}\langle-m,-p ; q\rangle_{i}\langle-m,-n ; q\rangle_{j} q^{j(a+m+n)}(-1)^{k+l}\langle-n,-p ; q\rangle_{k}\langle-n,-p ; q\rangle_{l}}{\langle 1, a ; q\rangle_{j}\left\langle 1, C_{2}-1, c_{2}+n, c_{2}+p ; q\right\rangle_{k}\langle 1, a+l-1, a+n, a+p ; q\rangle_{l}} \\
& \times \frac{\langle a, a ; q\rangle_{m}\left\langle a, b_{2} ; q\right\rangle_{n+p}\langle a ; q\rangle_{2 l}\left\langle c_{2} ; q\right\rangle_{2 k} q^{\left(\begin{array}{l}
k \\
2
\end{array}\right)+i(a+m+p)+k\left(c_{2}+n+p\right)+\left(\begin{array}{l}
l \\
2
\end{array}\right)+l(a+n+p)}}{\langle 1, a ; q\rangle_{i}\left\langle 1, c_{1} ; q\right\rangle_{m}\left\langle 1, c_{2} ; q\right\rangle_{n}\left\langle 1, c_{2} ; q\right\rangle_{p}} \\
& =\sum_{i j k l m n p} \frac{\langle 1, a, a ; q\rangle_{m} q^{i(a+i-1)+j(a+j-1)}\langle 1,1, a ; q\rangle_{n}\langle 1,1, a ; q\rangle_{p}(-1)^{k+l}\left\langle c_{2} ; q\right\rangle_{2 k}\langle a ; q\rangle_{2 l}}{\langle 1 ; q\rangle_{m-i}\langle 1 ; q\rangle_{m-j}\langle 1, a ; q\rangle_{i}\langle 1, a ; q\rangle_{j}\langle 1 ; q\rangle_{p-i}\langle 1, a+l-1, a, a ; q\rangle_{l}} \\
& \times \frac{X^{M}\left\langle a, b_{2} ; q\right\rangle_{n+p} q^{(3 / 2)\left(k^{2}-k\right)+k c_{2}+(3 / 2)\left(l^{2}-l\right)+l a}}{\langle 1 ; q\rangle_{n-j}\langle 1 ; q\rangle_{n-k}\langle 1 ; q\rangle_{n-l}\left\langle c_{1} ; q\right\rangle_{m}\left\langle a+l, C_{2} ; q\right\rangle_{n}\left\langle a+l, C_{2} ; q\right\rangle_{p}} \\
& \times \frac{1}{\langle 1 ; q\rangle_{p-k}\langle 1 ; q\rangle_{p-l}\left\langle 1, C_{2}-1, c_{2}, c_{2} ; q\right\rangle_{k}} \\
& \stackrel{\star}{=} \sum_{i j k l m n p} \frac{x_{1}^{m+i+j} x_{2}^{n+j+k+l} x_{3}^{p+i+k+l} q^{i(a+i-1)+j(a+j-1)+(3 / 2)\left(k^{2}-k\right)+k c_{2}+(3 / 2)\left(l^{2}-l\right)+l a}}{\langle 1 ; q\rangle_{p+k+l}\langle 1 ; q\rangle_{p+i+k}\langle 1, a ; q\rangle_{i}\langle 1, a ; q\rangle_{j}} \\
& \times \frac{\langle 1, a, a ; q\rangle_{m+i+j}\left\langle a, b_{2} ; q\right\rangle_{n+p+i+j+2 k+2 l}\langle a ; q\rangle_{2 l}}{\langle 1 ; q\rangle_{p+i+l}\langle 1 ; q\rangle_{n+l+k}\langle 1 ; q\rangle_{m+j}\langle 1 ; q\rangle_{m+i}\langle 1 ; q\rangle_{n+j+l}\left\langle c_{1} ; q\right\rangle_{m+i+j}\left\langle a+l, C_{2} ; q\right\rangle_{n+j+k+l}} \\
& \times \frac{\langle 1,1, a ; q\rangle_{n+j+k+l}\langle 1,1, a ; q\rangle_{p+i+k+l}(-1)^{k+l}\left\langle c_{2} ; q\right\rangle_{2 k}}{\langle 1 ; q\rangle_{n+j+k}\left\langle 1, C_{2}-1, c_{2}, c_{2} ; q\right\rangle_{k}\langle 1, a+l-1, a, a ; q\rangle_{l}\left\langle a+l, C_{2} ; q\right\rangle_{p+i+k+l}}=\text { RHS. }
\end{aligned}
$$

Put

$$
\begin{gathered}
\alpha \equiv(1,1+k+j, a+k+j, \infty, \infty) ; \\
(1,1+i+k, a+i+k, \infty, \infty),
\end{gathered}
$$




$$
\begin{aligned}
\beta \equiv & \left(a+j+2 k, c_{2}+j+k, 1+k, 1+j\right) ; \\
& \left(a+i+2 k, c_{3}+i+k, 1+i, 1+k\right) .
\end{aligned}
$$

Then,

$$
\begin{aligned}
& H_{B}\left(a, a, b_{2} ; c_{1}, c_{2}, c_{3} \mid q ; x_{1}, x_{2}, x_{3}\right) \\
& =\sum_{i j k} \frac{q^{i(a+i-1)+j(a+j-1)}\langle 1, a, a ; q\rangle_{i+j} x_{1}^{i+j} x_{2}^{k+j} x_{3}^{i+k} q^{(3 / 2)\left(k^{2}-k\right)+k a}}{\langle a+k ; q\rangle_{j+k}\langle a+k ; q\rangle_{i+k}\left\langle c_{2} ; q\right\rangle_{k+j}\left\langle c_{3} ; q\right\rangle_{i+k}} \\
& \quad \times \frac{\langle 1, a ; q\rangle_{k+j}\left\langle a, b_{2} ; q\right\rangle_{i+j+2 k}(-1)^{k}\langle a ; q\rangle_{2 k}\langle 1, a ; q\rangle_{i+k}\langle 1+i+k, a+i+k ; q\rangle_{p}}{\langle 1,1,1, a ; q\rangle_{i}\langle 1,1,1, a ; q\rangle_{j}\left\langle c_{1} ; q\right\rangle_{i+j}\langle 1,1, a+k-1, a, a ; q\rangle_{k}} \\
& \quad \times{ }_{4} \phi_{3}\left[\begin{array}{c}
1,1+i+j, a+i+j, a+i+j \\
1+i, 1+j, c_{1}+i+j
\end{array} \mid q ; x_{1}\right] \\
& \times \Phi_{2: 4}^{2: 5}\left[\begin{array}{c}
a+i+j+2 k, b_{2}+i+j+2 k: \alpha \\
\infty, \infty: \beta
\end{array} \mid q ; x_{2}, x_{3}\right] .
\end{aligned}
$$

Proof. Use formula (43). The computation goes as follows:

$$
\begin{aligned}
& H_{B}\left(a, a, b_{2} ; c_{1}, c_{2}, c_{3} \mid q ; x_{1}, x_{2}, x_{3}\right) \\
& =\sum_{i j k m n p} \frac{\langle-m,-p ; q\rangle_{i} q^{i(a+m+p)}\langle-m,-n ; q\rangle_{j} q^{j(a+m+n)}(-1)^{k}\langle-n,-p ; q\rangle_{k}\langle a ; q\rangle_{2 k}}{\langle 1, a ; q\rangle_{i}\langle 1, a ; q\rangle_{j}\langle 1, a+k-1, a+n, a+p ; q\rangle_{k}} \\
& \times X^{M} \frac{\langle a, a ; q\rangle_{m}\left\langle a, b_{2} ; q\right\rangle_{n+p}}{\left\langle 1, c_{1} ; q\right\rangle_{m}\left\langle 1, c_{2} ; q\right\rangle_{n}\left\langle 1, c_{3} ; q\right\rangle_{p}} q^{\left(\begin{array}{l}
k \\
2
\end{array}\right)+k(a+n+p)} \\
& =\sum_{i j k m n p} \frac{\langle 1, a, a ; q\rangle_{m} q^{i(a+i-1)+j(a+j-1)}\langle 1, a ; q\rangle_{p}(-1)^{k}\langle a ; q\rangle_{2 k}}{\left\langle c_{2} ; q\right\rangle_{n}\left\langle c_{3} ; q\right\rangle_{p}\langle 1 ; q\rangle_{m-i}\langle 1 ; q\rangle_{m-j}\langle 1, a ; q\rangle_{i}\langle 1, a ; q\rangle_{j}\langle 1 ; q\rangle_{p-i}} \\
& \times \frac{X^{M}\left\langle a, b_{2} ; q\right\rangle_{n+p}\langle a, 1 ; q\rangle_{n} q^{(3 / 2)\left(k^{2}-k\right)+k a}}{\langle 1 ; q\rangle_{n-j}\langle 1 ; q\rangle_{n-k}\left\langle c_{1} ; q\right\rangle_{m}\langle a+k ; q\rangle_{n}\langle a+k ; q\rangle_{p}\langle 1 ; q\rangle_{p-k}\langle 1, a+k-1, a, a ; q\rangle_{k}} \\
& \stackrel{\star}{=} \sum_{i j k m n p} \frac{q^{i(a+i-1)+j(a+j-1)}\langle 1, a, a ; q\rangle_{m+i+j} x_{1}^{m+i+j} x_{2}^{n+k+j} x_{3}^{p+i+k} q^{(3 / 2)\left(k^{2}-k\right)+k a}}{\left\langle a+k, c_{2} ; q\right\rangle_{n+j+k}\left\langle a+k, c_{3} ; q\right\rangle_{p+i+k}\langle 1, a ; q\rangle_{i}\langle 1, a ; q\rangle_{j}} \\
& \times \frac{\left\langle a, b_{2} ; q\right\rangle_{n+p+i+j+2 k}(-1)^{k}\langle a ; q\rangle_{2 k}}{\langle 1, a+k-1, a, a ; q\rangle_{k}} \\
& \times \frac{\langle 1, a ; q\rangle_{n+k+j}\langle 1, a ; q\rangle_{p+k+i}}{\langle 1 ; q\rangle_{m+j}\langle 1 ; q\rangle_{m+i}\langle 1 ; q\rangle_{p+k}\langle 1 ; q\rangle_{p+i}\langle 1 ; q\rangle_{n+k}\langle 1 ; q\rangle_{n+j}\left\langle c_{1} ; q\right\rangle_{m+i+j}}=\text { RHS. }
\end{aligned}
$$

Put

$$
\begin{gathered}
\alpha \equiv\left(1,1+k+j+l, 1+k+j+l, b_{1}\right. \\
\left.+k+j+l, b_{2}+k+j+l\right) ; \\
(1,1+i+k+l, 1+i+k+l, a \\
\left.+i+k+l, b_{2}+i+k+l\right)
\end{gathered}
$$




$$
\begin{aligned}
\beta \equiv & \left(c_{2}+j+2 k+l, 1+j+k, 1+k+l, 1+j+l\right) ; \\
& \left(c_{2}+i+2 k+l, 1+i+l, 1+k+l, 1+i+k\right) .
\end{aligned}
$$

Then,

$$
\begin{aligned}
& H_{A}\left(a, b_{1}, b_{2} ; c_{1}, c_{2} \mid q ; x_{1}, x_{2}, x_{3}\right) \\
& =\sum_{i j k l} \frac{q^{i(a+i-1)+j\left(b_{1}+j-1\right)+l\left(b_{2}+l-1\right)}\left\langle 1, a, b_{1} ; q\right\rangle_{i+j} x_{1}^{i+j} x_{2}^{k+j+l} x_{3}^{i+k+l} q^{(3 / 2)\left(k^{2}-k\right)+k c_{2}}}{\left\langle c_{2}+k ; q\right\rangle_{j+k+l}\left\langle c_{2}+k ; q\right\rangle_{i+k+l}\langle 1, a ; q\rangle_{i}} \\
& \quad \times \frac{\left\langle 1,1, b_{1}, b_{2} ; q\right\rangle_{k+j+l}\left\langle 1,1, a, b_{2} ; q\right\rangle_{i+k+l}(-1)^{k}\left\langle c_{2} ; q\right\rangle_{2 k}\langle 1, a ; q\rangle_{i+k}}{\left\langle 1, b_{2} ; q\right\rangle_{l}\left\langle 1,1, b_{1} ; q\right\rangle_{j}\left\langle c_{1} ; q\right\rangle_{i+j}\left\langle 1, c_{2}+k-1, c_{2}, c_{2} ; q\right\rangle_{k}} \\
& \quad \times \frac{1}{\langle 1,1, a ; q\rangle_{i}\langle 1 ; q\rangle_{j+k}\langle 1 ; q\rangle_{i+k}\langle 1 ; q\rangle_{i+l}\langle 1,1 ; q\rangle_{k+l}\langle 1 ; q\rangle_{j+l}} \\
& \quad \times{ }_{4} \phi_{3}\left[\begin{array}{c}
1,1+i+j, a+i+j, b_{1}+i+j \\
1+i, 1+j, c_{1}+i+j
\end{array} \mid q ; x_{1}\right] \Phi_{0: 4}^{0: 5}\left[\begin{array}{l}
-: \alpha \\
-: \beta
\end{array} \mid q ; x_{2}, x_{3}\right] .
\end{aligned}
$$

Proof. Use formula (45). The computation goes as follows:

$$
\begin{aligned}
& H_{A}\left(a, b_{1}, b_{2} ; c_{1}, c_{2} \mid q ; x_{1}, x_{2}, x_{3}\right) \\
&=\sum_{i j k l m n p} \frac{\langle-m,-p ; q\rangle_{i} q^{i(a+m+p)}\langle-m,-n ; q\rangle_{j} q^{j\left(b_{1}+m+n\right)}(-1)^{k}\langle-n,-p ; q\rangle_{k}\left\langle c_{2} ; q\right\rangle_{2 k}}{\langle 1, a ; q\rangle_{i}\left\langle 1, b_{1} ; q\right\rangle_{j}\left\langle 1, c_{2}+k-1, c_{2}+n, c_{2}+p ; q\right\rangle_{k}} \\
&\left.\times X^{M} \frac{\left\langle a, b_{1} ; q\right\rangle_{m}\left\langle b_{1}, b_{2} ; q\right\rangle_{n}\left\langle a, b_{2} ; q\right\rangle_{p}}{\left\langle 1, c_{1} ; q\right\rangle_{m}\left\langle 1, c_{2} ; q\right\rangle_{n}\left\langle 1, c_{2} ; q\right\rangle_{p}} q^{k}\right)+k\left(c_{2}+n+p\right) \frac{\langle-n,-p ; q\rangle_{l} q^{l\left(b_{2}+n+p\right)}}{\left\langle 1, b_{2} ; q\right\rangle_{l}} \\
&=\sum_{i j k l m n p} \frac{\left\langle 1, a, b_{1} ; q\right\rangle_{m} q^{i(a+i-1)+j\left(b_{1}+j-1\right)+l\left(b_{2}+l-1\right)}\left\langle 1,1, a, b_{2} ; q\right\rangle_{p}(-1)^{k}\left\langle c_{2} ; q\right\rangle_{2 k}}{\langle 1 ; q\rangle_{m-i}\langle 1 ; q\rangle_{m-j}\langle 1 ; q\rangle_{n-j}\langle 1 ; q\rangle_{n-k}\langle 1, a ; q\rangle_{i}\left\langle 1, b_{1} ; q\right\rangle_{j}\langle 1 ; q\rangle_{p-i}\left\langle 1, b_{2} ; q\right\rangle_{l}} \\
& \quad \times \frac{X^{M}\left\langle 1,1, b_{1}, b_{2} ; q\right\rangle_{n} q^{(3 / 2)\left(k^{2}-k\right)+k c_{2}}}{\langle 1 ; q\rangle_{n-l}\langle 1 ; q\rangle_{p-l}\left\langle c_{1} ; q\right\rangle_{m}\left\langle c_{2}+k ; q\right\rangle_{n}\left\langle c_{2}+k ; q\right\rangle_{p}\langle 1 ; q\rangle_{p-k}\left\langle 1, c_{2}+k-1, c_{2}, c_{2} ; q\right\rangle_{k}} \\
& \sum_{i j k l m n p} \frac{q^{i(a+i-1)+j\left(b_{1}+j-1\right)}\left\langle 1, a, b_{1} ; q\right\rangle_{m+i+j} x_{1}^{m+i+j} x_{2}^{n+j+k+l} x_{3}^{p+i+k+l} q^{(3 / 2)\left(k^{2}-k\right)+k c_{2}}}{\left\langle c_{2}+k ; q\right\rangle_{n+j+k+l}\left\langle c_{2}+k ; q\right\rangle_{p+i+k+l}\langle 1, a ; q\rangle_{i}\left\langle 1, b_{1} ; q\right\rangle_{j}} \\
& \quad \times \frac{\left\langle 1,1, b_{1}, b_{2} ; q\right\rangle_{n+k+j+l}}{\langle 1 ; q\rangle_{n+j+k}\langle 1 ; q\rangle_{p+i+k}\left\langle 1, b_{2} ; q\right\rangle_{l}\langle 1 ; q\rangle_{m+j}\langle 1 ; q\rangle_{m+i}\langle 1 ; q\rangle_{p+i+l}\langle 1 ; q\rangle_{n+k+l}\left\langle c_{1} ; q\right\rangle_{m+i+j}} \\
& \times \frac{\left\langle 1,1, a, b_{2} ; q\right\rangle_{p+i+k+l}(-1)^{k}\left\langle c_{2} ; q\right\rangle_{2 k} q^{l\left(b_{2}+l-1\right)}}{\langle 1 ; q\rangle_{n+j+l}\langle 1 ; q\rangle_{p+k+l}\left\langle 1, c_{2}+k-1, c_{2}, c_{2} ; q\right\rangle_{k}}=\mathrm{RHS.}
\end{aligned}
$$




$$
\begin{aligned}
& \alpha \equiv\left(1, b_{1}+j+k, 1+j+k, b_{2}+j+k\right) ;\left(1, a+i+k, 1+i+k, b_{2}+i+k\right) \\
& \beta \equiv\left(1+k, 1+j, c_{2}+j+k\right) ;\left(1+k, 1+i, c_{3}+i+k\right) .
\end{aligned}
$$

Then,

$$
\begin{aligned}
& H_{B}\left(a, b_{1}, b_{2} ; c_{1}, c_{2}, c_{3} \mid q ; x_{1}, x_{2}, x_{3}\right) \\
& =\sum_{i j k}\left\langle 1, a, b_{1} ; q\right\rangle_{i+j} Q E\left(j\left(b_{1}+j-1\right)\right) \frac{q^{i(a+i-1)+k\left(b_{2}+k-1\right)} x_{1}^{i+j} x_{2}^{j+k} x_{3}^{i+k} q\left\langle 1, a, b_{2} ; q\right\rangle_{i+k}\left\langle 1, b_{1}, b_{2} ; q\right\rangle_{j+k}}{\left\langle 1,1,1, b_{1} ; q\right\rangle_{j}\langle 1,1,1, a ; q\rangle_{i}\left\langle 1,1,1, b_{2} ; q\right\rangle_{k}\left\langle c_{1} ; q\right\rangle_{i+j}\left\langle c_{2} ; q\right\rangle_{j+k}\left\langle c_{3} ; q\right\rangle_{i+k}} \\
& \quad \times{ }_{4} \phi_{3}\left[\begin{array}{c}
1, a+i+j, b_{1}+i+j, 1+i+j \\
1+j, c_{1}+i+j, 1+i
\end{array} \quad \mid q ; x_{1}\right] \Phi_{0: 3}^{0: 4}\left[\begin{array}{c}
-: \alpha \\
-: \beta
\end{array} \mid q ; x_{2}, x_{3}\right] .
\end{aligned}
$$

Proof. Use formula (46). The computation goes as follows:

$$
\begin{aligned}
& H_{B}\left(a, b_{1}, b_{2} ; c_{1}, c_{2}, c_{3} \mid q ; x_{1}, x_{2}, x_{3}\right) \\
&= \sum_{i j k m n p} \frac{\langle-m,-p ; q\rangle_{i} q^{i(a+m+p)}\langle-m,-n ; q\rangle_{j} q^{j\left(b_{1}+m+n\right)}\langle-n,-p ; q\rangle_{k} q^{k\left(b_{2}+n+p\right)}}{\langle 1, a ; q\rangle_{i}\left\langle 1, b_{1} ; q\right\rangle_{j}\left\langle 1, b_{2} ; q\right\rangle_{k}} X^{M} \frac{\left\langle a, b_{1} ; q\right\rangle_{m}\left\langle b_{1}, b_{2} ; q\right\rangle_{n}\left\langle a, b_{2} ; q\right\rangle_{p}}{\left\langle 1, c_{1} ; q\right\rangle_{m}\left\langle 1, c_{2} ; q\right\rangle_{n}\left\langle 1, c_{3} ; q\right\rangle_{p}} \\
&= \sum_{i j k m n p} \frac{q^{i(a+i-1)+j\left(b_{1}+j-1\right)+k\left(b_{2}+k-1\right)}}{\langle 1 ; q\rangle_{m-i}\langle 1, a ; q\rangle_{i}\langle 1 ; q\rangle_{m-j}\langle 1 ; q\rangle_{p-i}\langle 1 ; q\rangle_{p-k}\left\langle 1, b_{1} ; q\right\rangle_{j}\left\langle 1, b_{2} ; q\right\rangle_{k}} X^{M} \frac{\left\langle 1, a, b_{1} ; q\right\rangle_{m}\left\langle 1, b_{1}, b_{2} ; q\right\rangle_{n}\left\langle 1, a, b_{2} ; q\right\rangle_{p}}{\langle 1 ; q\rangle_{n-j}\langle 1 ; q\rangle_{n-k}\left\langle c_{1} ; q\right\rangle_{m}\left\langle c_{2} ; q\right\rangle_{n}\left\langle c_{3} ; q\right\rangle_{p}} \\
& \quad \sum_{i j k m n p} \frac{\left\langle 1, a, b_{1} ; q\right\rangle_{i+j+m}\left\langle 1, a, b_{2} ; q\right\rangle_{i+k+p}\left\langle 1, b_{1}, b_{2} ; q\right\rangle_{j+k+n} x_{1}^{i+j+m}}{\langle 1 ; q\rangle_{m+j}\langle 1 ; q\rangle_{m+i}\langle 1 ; q\rangle_{k+p}\langle 1 ; q\rangle_{i+p}\langle 1, a ; q\rangle_{i}\left\langle 1, b_{1} ; q\right\rangle_{j}\left\langle 1, b_{2} ; q\right\rangle_{k}\langle 1 ; q\rangle_{j+n}} \\
& \quad \times \frac{x_{2}^{j+n+k} x_{3}^{i+k+p} q^{i(a+i-1)+j\left(b_{1}+j-1\right)+k\left(b_{2}+k-1\right)}}{\langle 1 ; q\rangle_{k+n}\left\langle c_{1} ; q\right\rangle_{i+j+m}\left\langle c_{2} ; q\right\rangle_{n+j+k}\left\langle c_{3} ; q\right\rangle_{p+i+k}}=\text { RHS. }
\end{aligned}
$$

The following two expansion formulas from [3] are special cases of our new results.

Theorem 9 (a q-analogue of Burchnall, Chaundy [5, (26)]). Consider

$$
\begin{aligned}
\Phi_{2}\left(a ; b, b^{\prime} ; c, c^{\prime} \mid q ; x_{1}, x_{2}\right) \\
=\sum_{r=0}^{\infty} \frac{\left\langle a, b, b^{\prime} ; q\right\rangle_{r}}{\left\langle 1, c, c^{\prime} ; q\right\rangle_{r}} x_{1}^{r} x_{2}^{r} q^{r(a+r-1)} \\
\quad \times{ }_{2} \phi_{1}\left(a+r, b+r ; c+r \mid q ; x_{1}\right) \\
\quad \times{ }_{2} \phi_{1}\left(a+r, b^{\prime}+r ; c^{\prime}+r \mid q ; x_{2}\right) .
\end{aligned}
$$

Theorem 10 (a q-analogue of $[5,(35)])$. Consider

$$
\begin{aligned}
& \Phi_{2}(\left.a ; b, b^{\prime} ; c, c \mid q ; x_{1}, x_{2}\right) \\
&= \sum_{r=0}^{\infty} \frac{\left\langle b, b^{\prime} ; q\right\rangle_{r}\langle a ; q\rangle_{2 r}}{\langle 1, c ; q\rangle_{r}\langle c ; q\rangle_{2 r}} q^{r c+r(r-1)} x_{1}^{r} x_{2}^{r} \\
& \quad \times \Phi_{1}\left(a+2 r ; b+r, b^{\prime}+r ; c+2 r \mid q ; x_{1}, x_{2}\right) .
\end{aligned}
$$

\section{Discussion}

To obtain the 10 new expansions for the three triple Srivastava hypergeometric functions in terms of ${ }_{4} F_{3}$ hypergeometric and Kampé de Fériet functions mentioned in the abstract, let 
all $\infty$ disappear and adapt the indices for $\Phi$ accordingly. All of this is explained in detail in the book [3].

We have not used the expansion formulas for $H_{C}$, since all these involve the operator $\widetilde{\Delta}_{x_{1} ; x_{2} x_{3} ; q}$, which has no simple summation formula. Expansion formulas for the first $q$ Lauricella function by the $\widetilde{\nabla}_{x_{1} ; x_{2} x_{3} ; q}$ operator will be given in the forthcoming paper.

We notice that the three triple $q$-functions have limits $q$-Appell functions and not $q$-Horn functions. The $q$-Horn functions will appear in another paper.

Hasanov, Srivastava, and Turaev have published several other articles of this type and Bin-Saad [2] treated the fourvariable case. The interested reader might consult papers by Exton on this last theme. Neither of these authors, however, used the $q$-operators from this paper.

The convergence regions for the $q$-Appell functions $\Phi_{2}$ and $\Phi_{4}$ given earlier are for the case $q=1$. For general $q$, the convergence regions are much larger as has been explained at the ICNAAM Conference 2010; see also [7].

\section{Conclusion}

The expansion formulas often have a symmetric form. If a number of $\infty$ appear up or down in the first place of the $\Phi$-function, an equal number of $\infty$ always appear opposite in the two second places of the $\Phi$-function. The sum of the number of indices of $q$-shifted factorials in numerator and denominator is always the same for every step in the proofs (except formula (70)). The simplest expansion is formula (50) and the most complicated one is (64). If a factor $(-1)$ appears, it comes from the operator $\Delta_{x_{1}, x_{2} ; q}$. The number of variables in the exponent in this case is equal to the number of $\Delta_{x_{1}, x_{2} ; q^{-}}$ operators.

\section{Conflict of Interests}

The author declares that there is no conflict of interests regarding the publication of this paper.

\section{References}

[1] A. Hasanov, H. M. Srivastava, and M. Turaev, "Decomposition formulas for some triple hypergeometric functions," Journal of Mathematical Analysis and Applications, vol. 324, no. 2, pp. 955969, 2006.

[2] M. G. Bin-Saad, "Symbolic operational images and decomposition formulas for hypergeometric functions," Journal of Mathematical Analysis and Applications, vol. 376, no. 2, pp. 451468, 2011.

[3] T. Ernst, A Comprehensive Treatment of $q$-Calculus, Birkhäuser, 2012.

[4] G. Gasper and M. Rahman, Basic Hypergeometric Series, Cambridge University Press, Cambridge, UK, 1990.

[5] J. L. Burchnall and T. W. Chaundy, "Expansions of appell's double hypergeometric functions," Quarterly Journal of Mathematics, vol. 11, no. 1, pp. 249-270, 1940.

[6] F. H. Jackson, "On basic double hypergeometric functions," Quarterly Journal of Mathematics, vol. 13, no. 1, pp. 69-82, 1942.
[7] T. Ernst, "Convergence aspects for q-Appell functions I," The Journal of the Indian Mathematical Society, vol. 81, no. 1-2, pp. 67-77, 2014. 


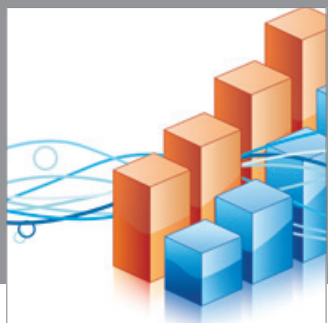

Advances in

Operations Research

mansans



The Scientific World Journal
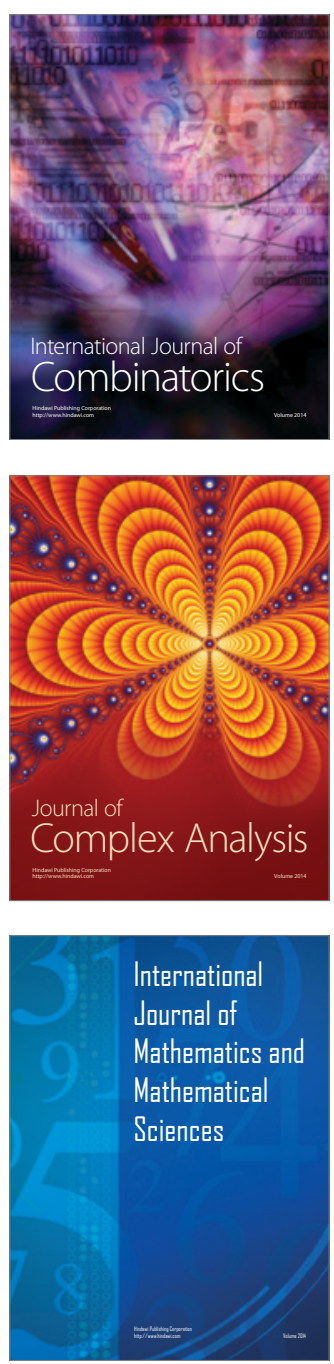
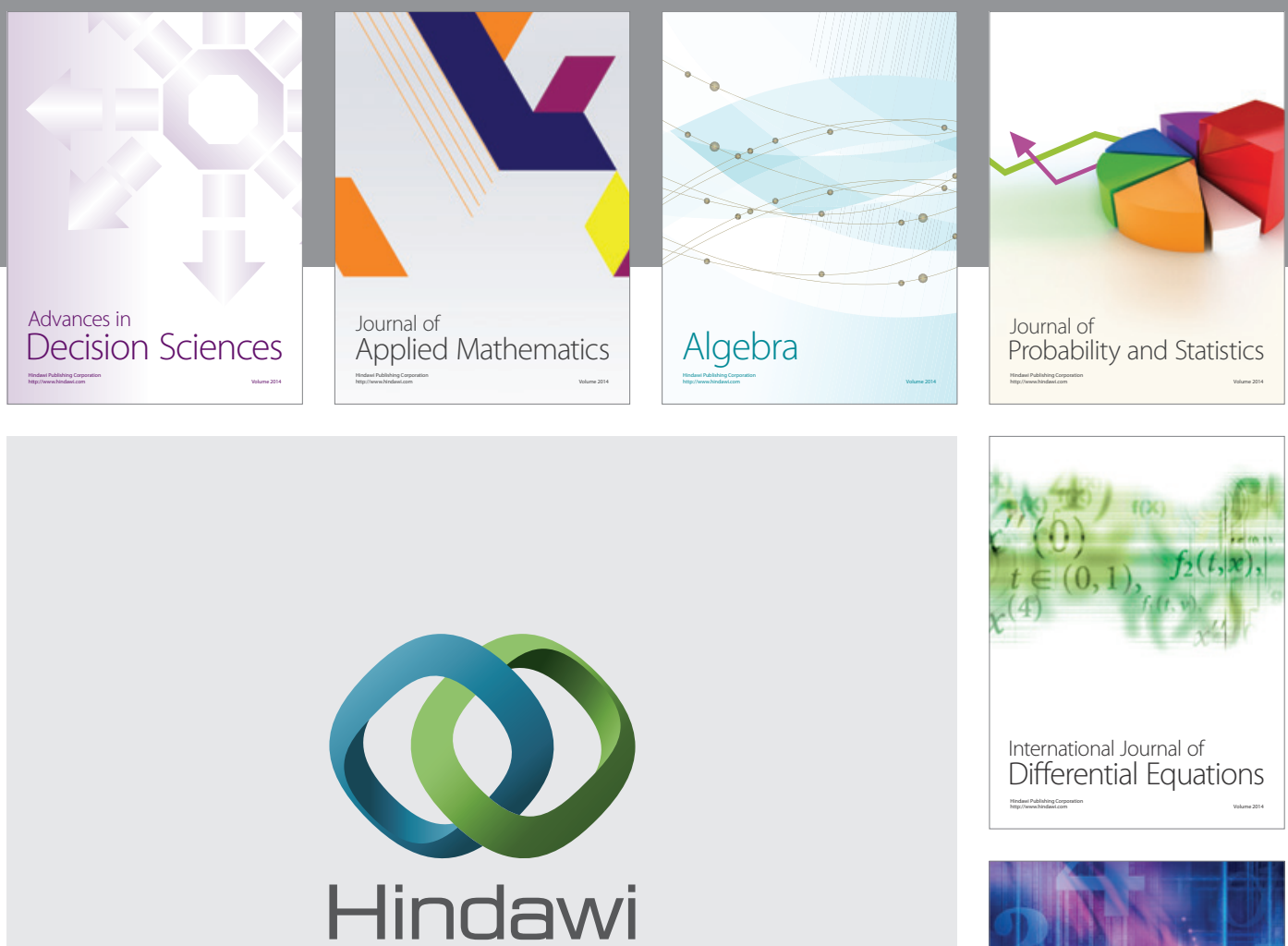

Submit your manuscripts at http://www.hindawi.com
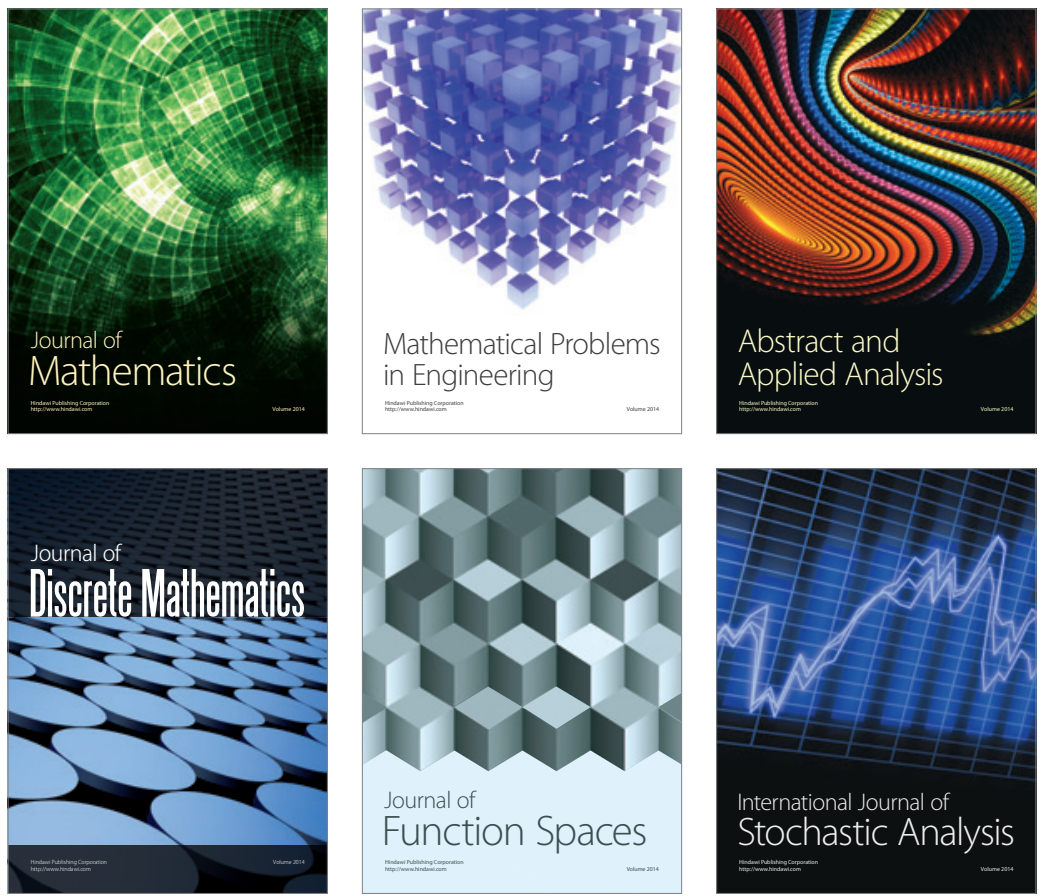

Journal of

Function Spaces

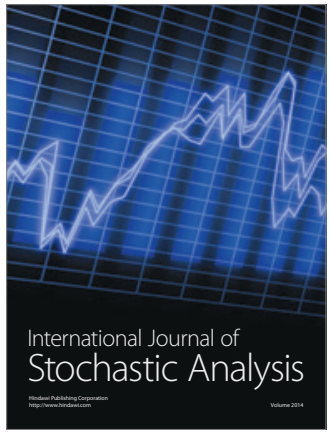

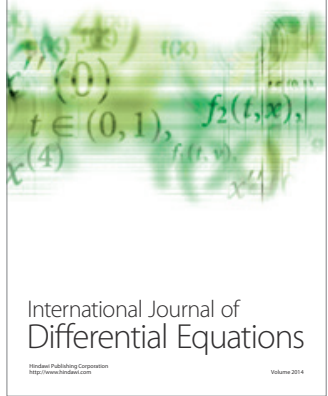
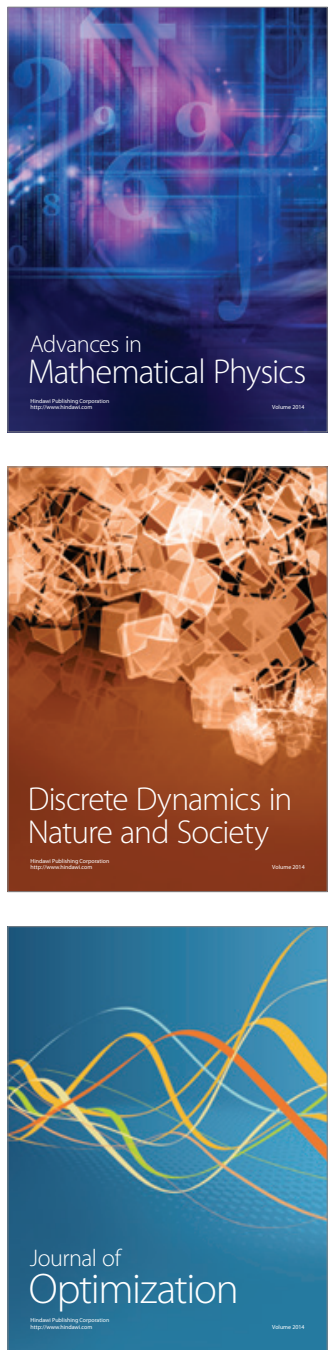